Supplement of Biogeosciences, 17, 3247-3275, 2020

https://doi.org/10.5194/bg-17-3247-2020-supplement

(C) Author(s) 2020. This work is distributed under

the Creative Commons Attribution 4.0 License.

(c) (1)

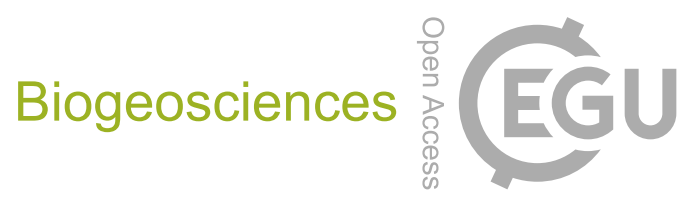

Supplement of

\title{
Assessing the potential for non-turbulent methane escape from the East Siberian Arctic Shelf
}

Matteo Puglini et al.

Correspondence to: Matteo Puglini (matteo.puglini@mpimet.mpg.de)

The copyright of individual parts of the supplement might differ from the CC BY 4.0 License. 


\section{S1 From continuity equation to advection-diffusion-reaction equation}

Each equation (one per species $i$ ) is a specific generalization of a continuity equation with fluxes $\mathscr{F}_{i}$, related to the the transport processes, and sources/sinks $\mathscr{S}_{i}$ related to the biogeochemical reactions. It reads:

$\frac{\partial \xi(z) C_{i}(z, t)}{\partial t}=-\frac{\partial \mathscr{F}_{i}(z, t)}{\partial z}+\mathscr{S}_{i}(z, t)$.

5 Where $C_{i}(z, t)$ is the concentration of the species $i$ (referred to porewater volume if it is a dissolved species or solid matrix volume if it is a solid species) and $\xi(z)$ is the term accounting for this, i.e. the porosity $\xi=\varphi$ in case of a dissolved species or the solid fraction $\xi=\varphi_{s}=1-\varphi$ in case of a solid species. The fluxes $\mathscr{F}_{i}(z, t)$ consist of several components:

- $\mathscr{F}_{D, i}(z, t)$ : the molecular diffusive flux (only for dissolved species) is given by the Fick's law

$$
\mathscr{F}_{D, i}(z, t)=-D_{i}(z, t) \varphi(z) \frac{\partial C_{i}(z, t)}{\partial z}
$$

where $D_{i}(z, t)$ is the effective diffusion coefficient. $D_{i}$ is usually modelled as a time-independent variable and considered constant for each species at a given salinity and temperature and locally only affected by sediment tortuosity $\theta(z)$. The relation between diffusion coefficient and salinity and temperature is provided in Table S6. Tortuosity has been considered strictly linked to porosity $\varphi(z)$ according to $\theta(z)=1-\ln \varphi^{2}(z)$. And porosity follows an exponential decay with depth (Athy, 1930):

$$
\varphi(z)=\varphi_{0} e^{-c_{0} z}
$$

with $\varphi_{0}$ porosity at the Sediment-Water Interface (SWI) and $c_{0}$ typical length scale for compaction (Table S6).

- $\mathscr{F}_{D_{b}, i}(z, t)$ : the bioturbation flux, described as a diffusive flux (to be considered also for solid species), reads

$$
\mathscr{F}_{D_{b}, i}(z, t)=-D_{b, i}(z, t) \xi(z) \frac{\partial C_{i}(z, t)}{\partial z}
$$

with $D_{b}(z, t)$ bioturbation coefficient. Considering no time dependency, the latter is assumed to follow an exponential trend below $5 \mathrm{~cm}$ depth, i.e. :

$$
\begin{cases}D_{b}(z)=D_{b}^{0}, & 0<=z<=5 \mathrm{~cm} \\ D_{b}(z)=D_{b}^{0} e^{-(z-5)}, & 5<z<300 \mathrm{~cm}\end{cases}
$$

and $D_{b}^{0}$ given in Table S6.

- $\mathscr{F}_{v, i}(z, t)$ : the advective flux is given by

$$
\mathscr{F}_{v, i}(z, t)=v(z, t) \xi(z) C_{i}(z, t)
$$

where $v(z, t)$ is the sedimentation rate $v(z, t)=\omega(z, t)$ for solid species and the sum of sedimentation rate and possible advective flow velocity $v_{u p}(z, t)$ for dissolved species, i.e. $v(z, t)=\omega(z, t)+v_{u p}(z, t)$. If we assume steady state compaction then burial velocity reads (Berner (1980)):

$\omega(z)=\left(\frac{1-\varphi_{0}}{1-\varphi(z)}\right) \omega_{0}$

with $\omega_{0}$ sedimentation rate at the SWI. A site where $v_{u p} \neq 0$ is denominated as active while a site with null upward water velocity is defined as passive. 
- $\mathscr{F}_{N L, i}(z, t)$ represent any form of non-local transport. In its more classical form (Boudreau (1997)) it is given by bioirrigation provided by irrigated furrows digged by benthic fauna and it reads

$$
\mathscr{F}_{i r r}(z, t)=-\int_{0}^{z} \alpha_{i}(y) \xi(y)\left[C_{i}(0, t)-C_{i}(y, t)\right] d y
$$

where $\alpha_{i}(z)$ is the bioirrigation coefficient, $C_{i}(0, t)$ is the concentration of the species $i$ at the SWI. Bioirrigation parameter $\alpha$ is modelled as (Thullner et al. (2009)):

$$
\alpha(z)=\alpha_{0} e^{-z / z_{i r r}}
$$

where $\alpha_{0}$ is the bioirrigation coefficient and $z_{i r r}$ the bioirrigation attenuation depth (see Table S6). Yet other forms of non-local transport may be considered in the Arctic shelf scenario, such as ice scouring or bubble migration in sediments rich of free gas. These processes are even more difficult to be modelled and are not included in current BRNS version.

With the fluxes described above eq. S1 takes the form of the standard advection-diffusion-reaction equation (see eq. 4) usually implemented in reactive-transport diagenetic models (Berner (1980); Boudreau (1997))

$20 \frac{\partial \xi C_{i}}{\partial t}=\frac{\partial}{\partial z}\left[\left(D_{i}+D_{b, i}\right) \xi \frac{\partial C_{i}}{\partial z}\right]-\frac{\partial}{\partial z}\left(v \xi C_{i}\right)+\alpha_{i} \xi\left(C_{i}(0)-C_{i}\right)+\mathscr{S}_{i}$.

\section{S2 Primary redox reactions}

Organic matter (OM) decomposition is a complex multi-step process (Arndt et al. (2013)) carried out by micro-organism along a chain of enzyme-mediated biochemical redox reactions which exploits electronic cascades to provide energy. Carbon in organic matter plays the role of electron donor, getting oxidized, and energy yield of the full redox reaction ultimately depends on the terminal electron acceptor (TEA) which overall acts as an oxidant, getting reduced. This entails a preferential sequence of how OM gets mineralized by microorganism along different metabolic pathways with different TEAs according to energy gain ladder.

It determines the typical vertical zonation of the TEAs and byproducts of organic matter decomposition throughout the sediment column according to the redox sequence (Claypool and Kaplan (1974); Froelich et al. (1979); Stumm and Morgan (1996)). The chained reactions involving OM mineralization are reported in Table S2. OM degradation is modeled by a generalized first order kinetic equation, namely decomposition rate is faster the more OM is present. The proportionality between organic carbon decay rate and organic carbon content is set by a degradability rate $k$ which not necessarily has to be constant. We employed reactive continuum model (RCM) to model this degradability rate $k$ as a continuous variable, whose distribution reads:

$g(k)=\frac{g_{0} k^{\nu-1} e^{-a k}}{\Gamma(\nu)}$

where $g_{0}$ is a scale parameter, $\nu$ and $a$ determine the shape of the distribution of $k$ and $\Gamma(\nu)$ is the Gamma function. The quantity $a$ represents the average lifetime of the more reactive organic matter components in a reactive-continuum model (Boudreau and Ruddick (1991)) and the mean degradation constant $\bar{k}$ of the OM spectrum is given by $\bar{k}=\nu / a$. The choice of RCM relies on the fact that it is considered more suitable for marine sediments than a discrete model (Aller and Blair (2004); Arndt et al. (2013)). It manages to encompass all the information about OM degradability in only two parameters, carriers of a theoretical meanings, instead that in as many degradation constants as carbon pools considered in a discrete model. Moreover RCM is more flexible to account for the whole range of degradability that Arctic OM shows, shifting its peak just by tweaking the parameters instead of the initial carbon content of each pool. Another peculiarity of this formulation of RCM is that, 
assuming distribution in eq. (S11), OM degradation can actually be rewritten as a first-order kinetic with the a degradability $K(t)$ dependent on sediment age $t$ (Boudreau and Ruddick (1991)), i.e.

$$
\mathscr{S}_{P O C}=\frac{d P O C(t)}{d t}=-K(t) P O C(t)=-\frac{\nu}{a+t} P O C(t)
$$

20 where $P O C(t)$ is the particulate organic carbon, which in turns reads

$$
P O C(t)=\operatorname{POC}(0)\left(\frac{a}{a+t}\right)^{\nu}
$$

$P O C(0)$ being the initial organic carbon concentration. This features allows to estimate sediment age and POC vertical profile just coupling RCM and a discrete model

\section{S3 Estimate of sediment age $t$ and POC content}

POC age and vertical profile are critical quantity to be evaluated. One approach would be performing ab initio simulations, but they are difficult since a complete knowledge of past boundary conditions and how they evolved over time would be needed. Even when this knowledge is enough, simulation times are usually rather long and hence a different approach is generally used. Using eq. (S7 and S3), in an unperturbed sediment setting, we find that the age at a certain depth $z$ is provided by

$t(z)=\int_{0}^{z} \frac{1}{\omega(z)}=\frac{z+\frac{\varphi_{0}}{c_{0}}\left(e^{-c_{0} z}-1\right)}{\omega_{0}\left(1-\varphi_{0}\right)}$.

If another formulation for the porosity is employed, for instance:

$\varphi(z)=\varphi_{\infty}+\left(\varphi_{0}-\varphi_{\infty}\right) e^{-c_{0} z}$

where $\varphi_{\infty}$ specifies the asymptotic value of the porosity, then the expression for the age $t(z)$ modifies as:

$t(z)=\int_{0}^{z} \frac{1}{\omega(z)}=\frac{\left(1-\varphi_{\infty}\right) z+\frac{\varphi_{0}-\varphi_{\infty}}{c_{0}}\left(e^{-c_{0} z}-1\right)}{\omega_{0}\left(1-\varphi_{0}\right)}$.

5 If the sediment column were not bioturbated, plugging results (S14) into eq. (S13) would give the steady state profile of the organic matter throughout the whole sediments, with $P O C(0)$ POC concentration at the SWI (because considerations above legitimize a space for time substitution and initial time corresponds to the uppermost concentration).

The column is instead divided in bioturbated (the upper part) and not-bioturbated (below a certain depth). Because of bioturbation the upper layers are mixed, i.e. POC of different ages coexist at the same depth (Meile and Van Cappellen (2005)).

10 This is actually the most difficult problem to be tackled to assign age to a certain sediment level. In order to deal with issue, similarly to (Dale et al., 2015), the POC in the bioturbated zone was modelled resorting to multi-G approximation of the RCM. It means that, within the bioturbated region, the POC is represented by 500 distinct $\mathrm{OM}$ fractions, whose reactivity covers the spectrum $k=\left[k_{\min }: 10^{-15}, k_{\max }:-\log (a)+2\right] \mathrm{yr}^{-1}$ plus two extra fractions accounting for the reactivity $\left[0, k_{\min }\right]$ and $\left[k_{\max }, \infty\right]$. With these considerations and considering constant porosity and sedimentation rate Eq. (S10) reads:

$\varphi_{s} \frac{\partial P O C(z, t)}{\partial t}=D_{b} \varphi_{s} \frac{\partial^{2} P O C(z, t)}{\partial z^{2}}-\omega \varphi_{s} \frac{\partial P O C(z, t)}{\partial z}-\varphi_{s} k_{i} P O C(z, t)$

where $\varphi_{s}=1-\varphi$ and $k_{i}$ is the reactivity of the $i$-th fraction of POC considered. Eq. (S17) is an advective-diffusive-reactive equation which in steady state can be analytically solved for each fraction $i$, once boundary conditions are provided. The 
general solution is:

$\operatorname{POC}(z)=A e^{\lambda_{1} z}+B e^{\lambda_{2} z}$ where $\left\{\begin{array}{l}\lambda_{1}=\frac{\omega-\sqrt{\omega^{2}+4 D_{b} k_{i}}}{2 D_{b}} \\ \lambda_{2}=\frac{\omega+\sqrt{\omega^{2}+4 D_{b} k_{i}}}{2 D_{b}} .\end{array}\right.$

20 In this case boundary conditions are given by

$$
\begin{cases}P O C(0)=P O C_{0} \cdot F_{i} & \text { at } z=0 \\ D_{b} \frac{\partial P O C}{\partial z}=0 & \text { at } z=z_{b i o}\end{cases}
$$

where $F_{i}$ is the fraction of initial $P O C_{0}$ whose reactivity lies around $k_{i}$ and $z_{b i o}$ is the depth of bioturbated zone where we impose that bioturbated flux shall be null. Bearing in mind eq. (S12) and (S13), the distribution of the $P O C_{0}$ lying around a certain value of $k=k_{i}$ can be written as:

$25 f\left(k_{i}\right)=\frac{g\left(k_{i}\right)}{P O C_{0}}=\frac{a^{\nu} k_{i}^{\nu-1} e^{-a k_{i}}}{\Gamma(\nu)}$.

The initial fraction of $P O C_{0}$ whose reactivity is within $\left[0, k_{i}\right]$, i.e. $F\left(k_{i}\right)$, can be obtained via integration of eq. (S20) and this allows to find finally the fraction $F_{i}$ of $P O C_{0}$ whose reactivity is in $\left[k_{i-1}, k_{i}\right]$ as

$F_{i}=F\left(k_{i}\right)-F\left(k_{i-1}\right)$.

Enforcing boundary conditions (S19) for each of 502 fractions and summing up their solutions we find an approximate solution 5 of the RCM in the bioturbated layer. A comparison between the solution obtained in this way and what should be the RCM solution (eq. (S13)) allows to find the apparent age $t(z)$ of the sediments at each depth within the bioturbated zone and also at its bottom, i.e. $t\left(z_{b i o}\right)$. Below the bioturbated zone the apparent age is then found simply adding $t\left(z_{b i o}\right)$ and results of eq. (S14).

The age profile and the POC profile found in this way is then calculated from BRNS at the very beginning and imposed throughout the whole steady-state simulation. 


\section{S4 Biogeochemical network: tables}

Table S1. The 6 inhibition factors ruling the onset of metabolic pathways according to the succession of the redox ladder.

\begin{tabular}{|c|c|}
\hline $\mathrm{f}_{\mathrm{O}_{2}}$ & $\begin{array}{ll}1 & \text { if }\left[\mathrm{O}_{2}\right]>K_{\mathrm{O}_{2}} \\
\frac{\left[\mathrm{O}_{2}\right]}{K_{\mathrm{O}_{2}}} & \text { if }\left[\mathrm{O}_{2}\right]<K_{\mathrm{O}_{2}}\end{array}$ \\
\hline $\mathrm{f}_{\mathrm{NO}_{3}}{ }^{-}$ & $\begin{array}{ll}0 & \text { if }\left[\mathrm{O}_{2}\right]>K_{\mathrm{O}_{2}} \\
\left(1-\frac{\left[\mathrm{O}_{2}\right]}{K_{\mathrm{O}_{2}}}\right) \frac{\mathrm{NO}_{3}{ }^{-}}{K_{\mathrm{NO}_{3}-}^{-}} & \text {if }\left[\mathrm{O}_{2}\right]<K_{\mathrm{O}_{2}}\end{array}$ \\
\hline $\mathrm{f}_{\mathrm{MnO}_{2}}$ & $\begin{array}{ll}0 & \text { if }\left[\mathrm{NO}_{3}^{-}\right]>K_{\mathrm{NO}_{3}}{ }^{-} \\
\left(1-\frac{\left[\mathrm{O}_{2}\right]}{K_{\mathrm{O}_{2}}}-\frac{\left[\mathrm{NO}_{3}^{-}\right]}{K_{\mathrm{NO}_{3}}^{-}}\right) \frac{\mathrm{MnO}_{2}}{K_{\mathrm{MnO}_{2}}} & \text { if }\left[\mathrm{NO}_{3}^{-}\right]<K_{\mathrm{NO}_{3}}^{-}\end{array}$ \\
\hline $\mathrm{f}_{\mathrm{Fe}(\mathrm{OH})_{3}}$ & $\begin{array}{ll}0 & \text { if }\left[\mathrm{MnO}_{2}\right]>K_{\mathrm{MnO}_{2}} \\
\left(1-\frac{\left[\mathrm{O}_{2}\right]}{K_{\mathrm{O}_{2}}}-\frac{\left[\mathrm{NO}_{3}^{-}\right]}{K_{\mathrm{NO}_{3}}{ }^{-}}-\frac{\left[\mathrm{MnO}_{2}\right]}{K_{\mathrm{MnO}_{2}}}\right) \frac{\mathrm{Fe}(\mathrm{OH})_{3}}{K_{\mathrm{Fe}(\mathrm{OH})_{3}}} & \text { if }\left[\mathrm{MnO}_{2}\right]<K_{\mathrm{MnO}_{2}}\end{array}$ \\
\hline $\mathrm{f}_{\mathrm{SO}_{4}{ }^{2-}}$ & $\begin{array}{ll}0 & \text { if }\left[\mathrm{Fe}(\mathrm{OH})_{3}\right]>K_{\mathrm{Fe}(\mathrm{OH})_{3}} \\
\left(1-\frac{\left[\mathrm{O}_{2}\right]}{K_{\mathrm{O}_{2}}}-\frac{\left[\mathrm{NO}_{3}{ }^{-}\right]}{K_{\mathrm{NO}_{3}{ }^{-}}}-\frac{\left[\mathrm{MnO}_{2}\right]}{K_{\mathrm{MnO}_{2}}}-\frac{\left[\mathrm{Fe}(\mathrm{OH})_{3}\right]}{K_{\mathrm{Fe}(\mathrm{OH})_{3}}}\right) \frac{\mathrm{SO}_{4}{ }^{2-}}{K_{\mathrm{SO}_{4}{ }^{2-}}} & \text { if }\left[\mathrm{Fe}(\mathrm{OH})_{3}\right]<K_{\mathrm{Fe}(\mathrm{OH})_{3}}\end{array}$ \\
\hline $\mathrm{f}_{\mathrm{CH}_{4}}$ & $\begin{array}{ll}0 & \text { if }\left[\mathrm{SO}_{4}{ }^{2-}\right]>K_{\mathrm{SO}_{4}{ }^{2-}} \\
\left(1-\frac{\left[\mathrm{O}_{2}\right]}{K_{\mathrm{O}_{2}}}-\frac{\left[\mathrm{NO}_{3}{ }^{-}\right]}{K_{\mathrm{NO}_{3}{ }^{-}}}-\frac{\left[\mathrm{MnO}_{2}\right]}{K_{\mathrm{MnO}_{2}}}-\frac{\left[\mathrm{Fe}(\mathrm{OH})_{3}\right]}{K_{\mathrm{Fe}(\mathrm{OH})_{3}}}-\frac{\left[\mathrm{SO}_{4}{ }^{2-}\right]}{K_{\mathrm{SO}_{4}}{ }^{2-}}\right) \frac{\mathrm{CH}_{4}}{K_{\mathrm{CH}_{4}}} & \text { if }\left[\mathrm{SO}_{4}{ }^{2-}\right]<K_{\mathrm{SO}_{4}{ }^{2-}}\end{array}$ \\
\hline
\end{tabular}




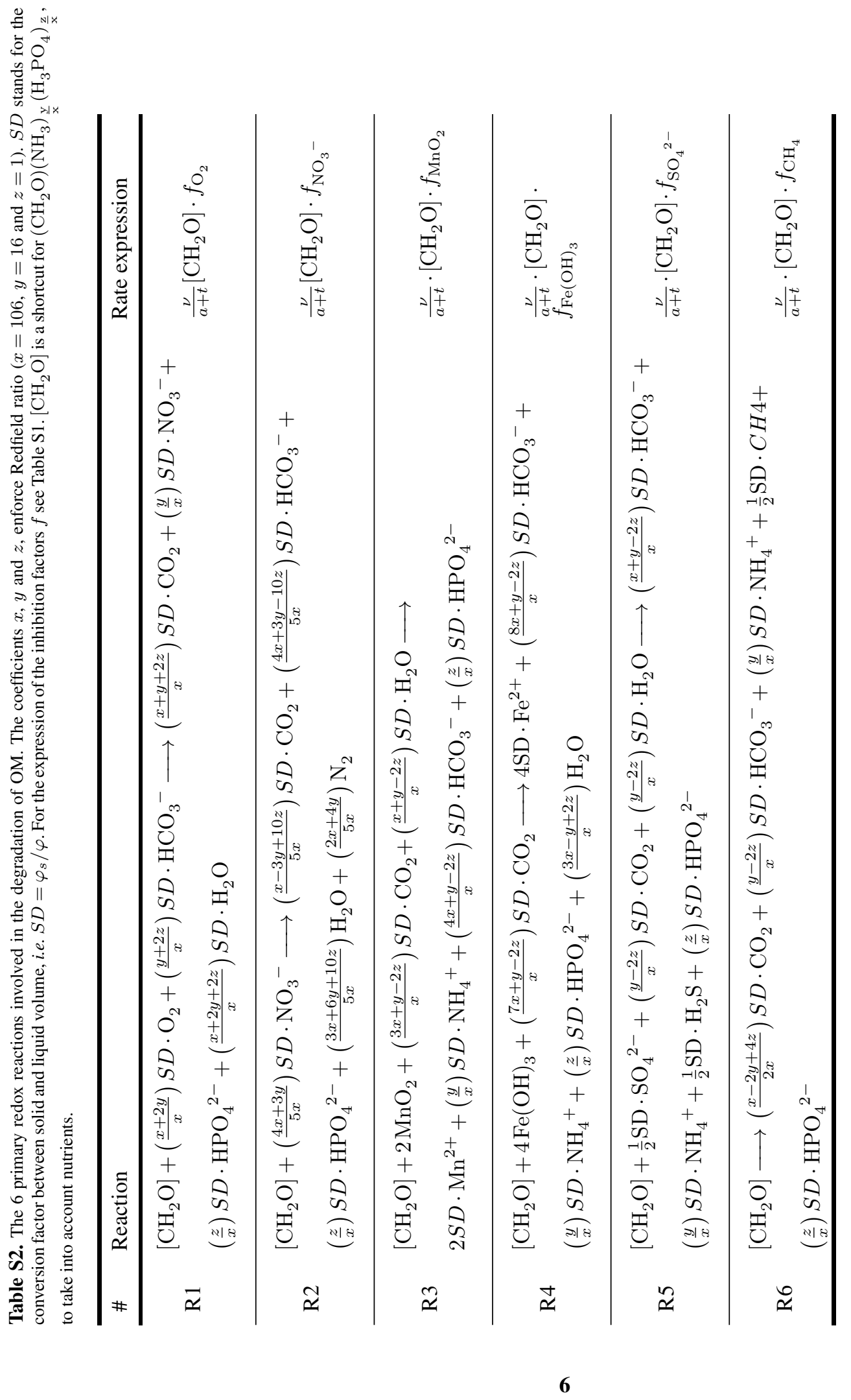




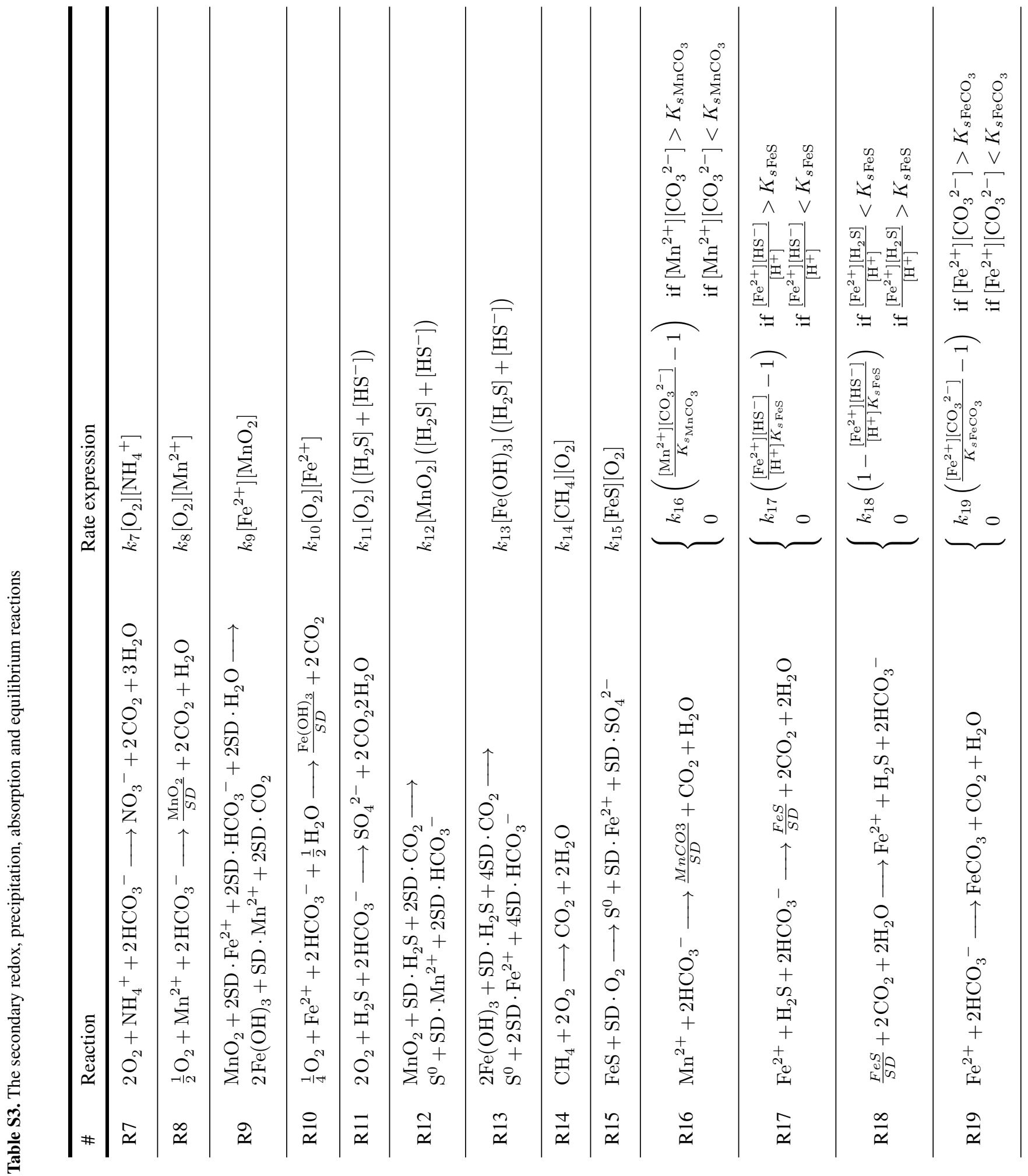




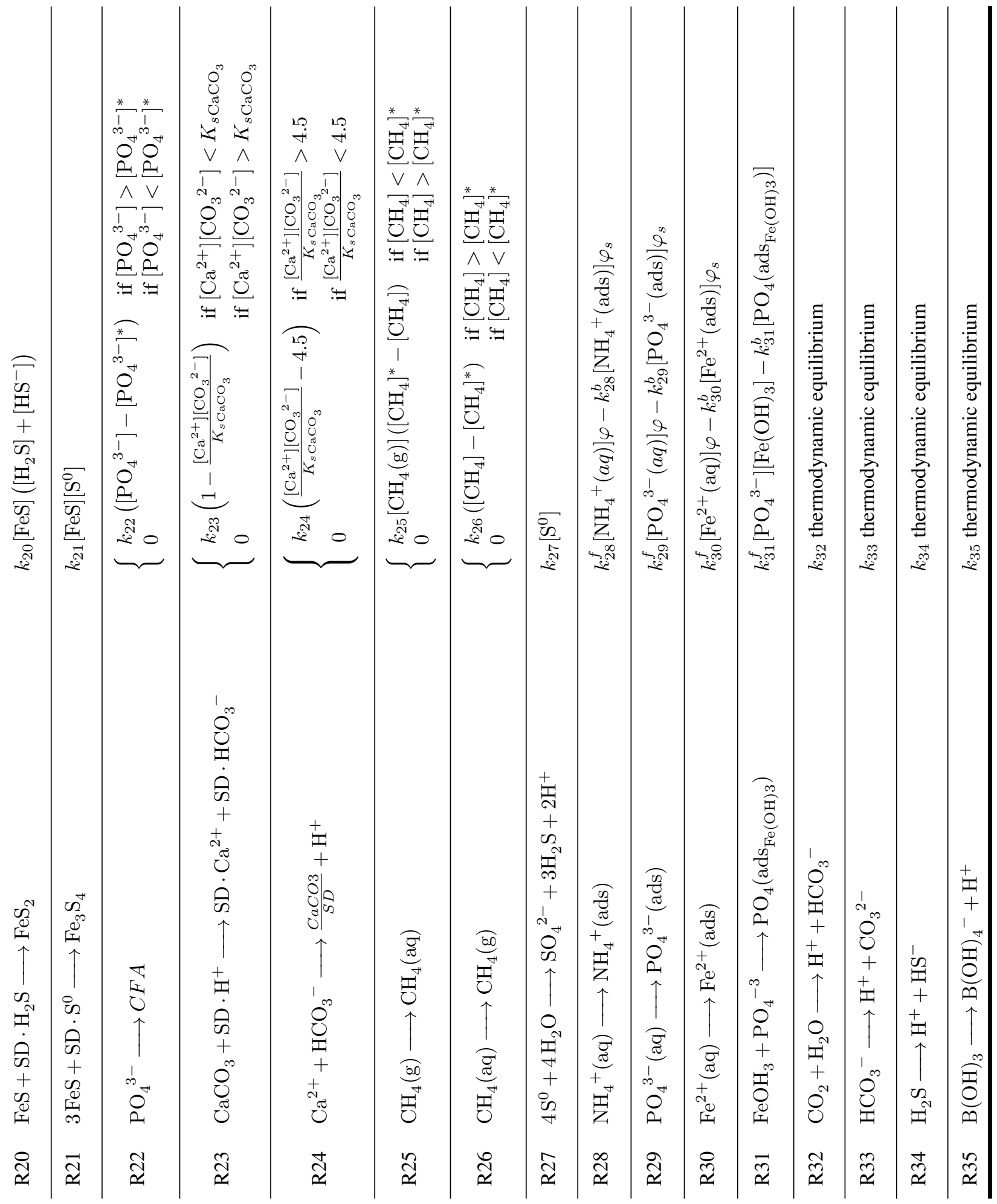




\section{S5 Model validations}

\section{S5.1 Sediment core offshore the Laptev Sea}

Table S4. Model boundary parameters and boundary conditions for the case study of a site offshore the Laptev Sea, Russia (core 14-3). Values with (!) are set for this study. All the other variables are as in Brüchert et al. (2018) and Brüchert (2020). [] - stands for lower boundary 5 conditions (at $3 \mathrm{~m} \mathrm{depth}$ ), while [ ] stand for upper boundary conditions. "Extrapolation" means that the value has been extrapolated from data.

\begin{tabular}{lll}
\hline Quantity & Value & Units \\
\hline$a(!)$ & 10 & $\mathrm{yr}$ \\
$\nu(!)$ & 0.200 & - \\
$\left.v_{u p} \mathrm{SO}_{4}{ }^{2-}\right]_{+}$ & 0.0 & $\mathrm{~cm} \mathrm{yr}^{-1}$ \\
{$\left[\mathrm{O}_{2}\right]_{+}$} & 27.95 & $\mathrm{mM}$ \\
{$\left[\mathrm{CH}_{4}\right]_{+}$} & 326.61 & $\mu \mathrm{M}$ \\
{$\left[\mathrm{CH}_{4}\right]_{-}(!)$} & 0.0 & $\mathrm{nM}$ \\
{$\left[\mathrm{CH}_{4}\right]^{*}$} & 16.0 & $\mathrm{mM}$ \\
{$\left[\mathrm{NH}_{4}{ }^{+}\right]_{+}$} & 14.0 & $\mathrm{mM}$ \\
$k_{A O M}(!)$ & 8.0 & $\mu \mathrm{M}$ (extrapolation) \\
$k_{28}^{f}(!)$ & $1.0 \cdot 10^{4}$ & $\mathrm{M}^{-1} \mathrm{yr}^{-1}$ \\
{$\left[\mathrm{PO}_{4}{ }^{3-}\right]^{*}(!)$} & 500 & $\mathrm{yr}^{-1}$ \\
\hline
\end{tabular}


Table S5. Organic carbon content (Brüchert, 2020) for a site offshore the Laptev Sea, Russia (core 14-3). The average density of the sediment matrix is $2.76 \mathrm{~g} \mathrm{~cm}^{-3}$ (Brüchert et al., 2018).

\begin{tabular}{ll}
\hline Depth $[\mathrm{cm}]$ & Organic Carbon content [\%] \\
\hline 3 & 0.88 \\
5 & 0.29 \\
7 & 0.51 \\
9 & 0.84 \\
11 & 0.59 \\
14.5 & 0.72 \\
17 & 0.74 \\
19 & 0.4 \\
\hline
\end{tabular}

\section{S6 Sensitivity study: model parameters and model boundary conditions. Tables}

Table S6. Generic model parameters. The parameters below the red line at the bottom are the quantities involved in the bioenergetic formulation of AOM. Diffusion coefficients depends on temperature $T\left({ }^{\circ} \mathrm{C}\right)$, salinity $S$ and porosity $\varphi$ according to the relation $D(T, S, \varphi)=\frac{D\left(1+\mu T\left({ }^{\circ} C\right)\right)}{1-\ln \left(\varphi^{2}\right)}$.

\begin{tabular}{|c|c|c|c|}
\hline Quantity & Value & Units & Reference \\
\hline$\nu$ & 0.125 & - & This study \\
\hline SD & $\varphi_{s} / \varphi$ & - & Athy (1930) \\
\hline$S$ & 20 & psu & This study \\
\hline$\rho$ & 2.41 & $\mathrm{~g} \mathrm{~cm}^{-3}$ & Berg (2003) \\
\hline$D_{b}^{0}$ & 29.8 & $\mathrm{~cm}^{2} \mathrm{yr}^{-1}$ & Middelburg et al. (1997) \\
\hline$z_{b i o}$ & 5 & $\mathrm{~cm}^{2} \mathrm{yr}^{-1}$ & Boudreau (1997) \\
\hline$z_{i r r}$ & 3.5 & $\mathrm{~cm}$ & Thullner et al. (2009) \\
\hline$T$ & $0,273.15$ & ${ }^{\circ} \mathrm{C}, \mathrm{K}$ & This study \\
\hline $\mathrm{C}: \mathrm{N}$ & $106: 16$ & - & Redfield ratio \\
\hline $\mathrm{C}: \mathrm{P}$ & $106: 1$ & - & Redfield ratio \\
\hline$D_{\mathrm{O}_{2}}, \mu_{\mathrm{O}_{2}}$ & $380.45,0.06$ & $\mathrm{~cm}^{2} \mathrm{yr}^{-1}, \mathrm{~T}^{-1}$ & Dale et al. (2012) \\
\hline$D_{\mathrm{NO}_{3}-}^{-}, \mu_{\mathrm{NO}_{3}-}^{-}$ & $394.59,0.038$ & $\mathrm{~cm}^{2} \mathrm{yr}^{-1}, \mathrm{~T}^{-1}$ & Van Cappellen and Wang (1996) \\
\hline 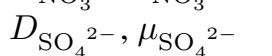 & $173.92,0.045$ & $\mathrm{~cm}^{2} \mathrm{yr}^{-1}, \mathrm{~T}^{-1}$ & Dale et al. (2012) \\
\hline$D_{\mathrm{CH}_{4}}, \mu_{\mathrm{CH}_{4}}$ & $263.94,0.052$ & $\mathrm{~cm}^{2} \mathrm{yr}^{-1}, \mathrm{~T}^{-1}$ & Van Cappellen and Wang (1996) \\
\hline$D_{\mathrm{NH}_{4}^{+}}, \mu_{\mathrm{NH}_{4}^{+}}^{+}$ & $395.87,0.041$ & $\mathrm{~cm}^{2} \mathrm{yr}^{-1}, \mathrm{~T}^{-1}$ & Van Cappellen and Wang (1996) \\
\hline 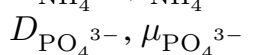 & $112.36,0.054$ & $\mathrm{~cm}^{2} \mathrm{yr}^{-1}, \mathrm{~T}^{-1}$ & Van Cappellen and Wang (1996) \\
\hline$D_{\mathrm{Mn}^{2+}, \mu_{\mathrm{Mn}^{2+}}}$ & $123.39,0.05$ & $\mathrm{~cm}^{2} \mathrm{yr}^{-1}, \mathrm{~T}^{-1}$ & Van Cappellen and Wang (1996) \\
\hline$D_{\mathrm{Fe}^{2+}}, \mu_{\mathrm{Fe}^{2+}}$ & $136.24,0.044$ & $\mathrm{~cm}^{2} \mathrm{yr}^{-1}, \mathrm{~T}^{-1}$ & Dale et al. (2012) \\
\hline$D_{\mathrm{H}_{2} \mathrm{~S}}, \mu_{\mathrm{H}_{2} \mathrm{~S}}$ & $331.61,0.06$ & $\mathrm{~cm}^{2} \mathrm{yr}^{-1}, \mathrm{~T}^{-1}$ & Van Cappellen and Wang (1996) \\
\hline$D_{\mathrm{HS}^{-}}, \mu_{\mathrm{HS}^{-}}$ & $392.02,0.031$ & $\mathrm{~cm}^{2} \mathrm{yr}^{-1}, \mathrm{~T}^{-1}$ & Van Cappellen and Wang (1996) \\
\hline$D_{\mathrm{CH}_{4}(\mathrm{~g})}, \mu_{\mathrm{CH}_{4}(\mathrm{~g})}$ & $5000.0,0.0$ & $\mathrm{~cm}^{2} \mathrm{yr}^{-1}, \mathrm{~T}^{-1}$ & Van Cappellen and Wang (1996) \\
\hline 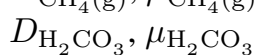 & $320.04,0.06$ & $\mathrm{~cm}^{2} \mathrm{yr}^{-1}, \mathrm{~T}^{-1}$ & Van Cappellen and Wang (1996) \\
\hline$D_{\mathrm{HCO}_{3}}^{-}, \mu_{\mathrm{HCO}_{3}}^{-}$ & $217.22,0.048$ & $\mathrm{~cm}^{2} \mathrm{yr}^{-1}, \mathrm{~T}^{-1}$ & Van Cappellen and Wang (1996) \\
\hline
\end{tabular}




\begin{tabular}{l}
$D_{\mathrm{CO}_{3}{ }^{2-}}, \mu_{\mathrm{CO}_{3}{ }^{2-}}$ \\
$D_{\mathrm{B}(\mathrm{OH})_{3}}, \mu_{\mathrm{B}(\mathrm{OH})_{3}}$ \\
$D_{\mathrm{B}(\mathrm{OH})_{4}}, \mu_{\mathrm{B}(\mathrm{OH})_{4}}{ }^{-}$ \\
$D_{\mathrm{H}^{+}}, \mu_{\mathrm{H}^{+}}$ \\
$D_{\mathrm{Ca}^{2+}, \mu_{\mathrm{Ca}^{2+}}}$ \\
$D_{\mathrm{S}^{0}}, \mu_{\mathrm{S}^{0}}$ \\
$\varphi_{0}$ \\
$c_{0}$ \\
$\varphi_{\infty}$ \\
$K_{\mathrm{O}_{2}}$ \\
$K_{\mathrm{NO}_{3}}{ }^{-}$ \\
$K_{\mathrm{MnO}_{2}}$ \\
$K_{\mathrm{Fe} \mathrm{OH})_{3}}$ \\
$K_{\mathrm{SO}_{4}}{ }^{2-}$ \\
$k_{7}$ \\
$k_{8}$ \\
$k_{9}$ \\
$k_{10}$ \\
$k_{11}$ \\
$k_{12}$ \\
$k_{13}$ \\
$k_{14}$ \\
$k_{15}$ \\
$k_{16}$ \\
$k_{17}$ \\
$k_{18}$ \\
$k_{19}$ \\
$k_{20}$ \\
$k_{21}$ \\
$k_{22}$ \\
$k_{23}$ \\
$k_{24}$ \\
$k_{25}$ \\
$k_{26}$ \\
$k_{27}$ \\
$k_{28}^{f}$ \\
$k_{28}^{b}$ \\
$k_{29}^{f}$ \\
$k_{29}^{b}$ \\
$k_{30}^{f}$ \\
$k_{30}^{b}$ \\
$k_{31}^{f}$ \\
$k_{31}^{b}$ \\
$k_{32}^{b}$ \\
$k_{33}$ \\
$k_{34}$ \\
\\
\hline
\end{tabular}

\begin{tabular}{|c|c|}
\hline $176.09,0.047$ & $\mathrm{~cm}^{2} \mathrm{yr}^{-1}, \mathrm{~T}^{-1}$ \\
\hline $110.05,0.054$ & $\mathrm{~cm}^{2} \mathrm{yr}^{-1}, \mathrm{~T}^{-1}$ \\
\hline $96.30,0.041$ & $\mathrm{~cm}^{2} \mathrm{yr}^{-1}, \mathrm{~T}^{-1}$ \\
\hline $600.0,0.05$ & $\mathrm{~cm}^{2} \mathrm{yr}^{-1}, \mathrm{~T}^{-1}$ \\
\hline $150.38,0.045$ & $\mathrm{~cm}^{2} \mathrm{yr}^{-1}, \mathrm{~T}^{-1}$ \\
\hline $173.92,0.045$ & $\mathrm{~cm}^{2} \mathrm{yr}^{-1}, \mathrm{~T}^{-1}$ \\
\hline 0.45 & - \\
\hline $0.5 \cdot 10^{-3}$ & $\mathrm{~m}^{-1}$ \\
\hline 0.35 & - \\
\hline 8 & $\mu \mathrm{M}$ \\
\hline 5 & $\mu \mathrm{M}$ \\
\hline 2 & $\mu \mathrm{mol} / \mathrm{g}_{d}$ \\
\hline 5 & $\mu \mathrm{mol} / \mathrm{g}_{d}$ \\
\hline 100 & $\mu \mathrm{M}$ \\
\hline $1.0 \cdot 10^{7}$ & $\mathrm{~cm}^{3} \mathrm{~mol}^{-1} \mathrm{yr}^{-1}$ \\
\hline $2.0 \cdot 10^{9}$ & $\mathrm{M}^{-1} \mathrm{yr}^{-1}$ \\
\hline $2.0 \cdot 10^{8}$ & $\mathrm{M}^{-1} \mathrm{yr}^{-1}$ \\
\hline $1.0 \cdot 10^{11}$ & $\mathrm{~cm}^{3} \mathrm{~mol}^{-1} \mathrm{yr}^{-1}$ \\
\hline $1.0 \cdot 10^{9}$ & $\mathrm{~cm}^{3} \mathrm{~mol}^{-1} \mathrm{yr}^{-1}$ \\
\hline $1.0 \cdot 10^{4}$ & $\mathrm{M}^{-1} \mathrm{yr}^{-1}$ \\
\hline $1.0 \cdot 10^{4}$ & $\mathrm{M}^{-1} \mathrm{yr}^{-1}$ \\
\hline $1.0 \cdot 10^{13}$ & $\mathrm{~cm}^{3} \mathrm{~mol}^{-1} \mathrm{yr}^{-1}$ \\
\hline $1.0 \cdot 10^{9}$ & $\mathrm{~cm}^{3} \mathrm{~mol}^{-1} \mathrm{yr}^{-1}$ \\
\hline $1.0 \cdot 10^{-8}$ & $\mathrm{~mol} \mathrm{~cm}^{-3} \mathrm{yr}^{-1}$ \\
\hline $5 \cdot 10^{-9}$ & $\mathrm{~mol} \mathrm{~cm}{ }^{-3} \mathrm{yr}^{-1}$ \\
\hline $1.0 \cdot 10^{-3}$ & $\mathrm{~mol} \mathrm{~cm}^{-3} \mathrm{yr}^{-1}$ \\
\hline $1.0 \cdot 10^{-9}$ & $\mathrm{~mol} \mathrm{~cm}^{-3} \mathrm{yr}^{-1}$ \\
\hline $6.0 \cdot 10^{7}$ & $\mathrm{~cm}^{3} \mathrm{~mol}^{-1} \mathrm{yr}^{-1}$ \\
\hline $2.4 \cdot 10^{4}$ & $\mathrm{~cm}^{3} \mathrm{~mol}^{-1} \mathrm{yr}^{-1}$ \\
\hline 1 & $\mathrm{yr}^{-1}$ \\
\hline 0.1 & $\mathrm{~mol} \mathrm{~cm}{ }^{-3} \mathrm{yr}^{-1}$ \\
\hline 24.16 & $\mathrm{M}^{-1} \mathrm{yr}^{-1}$ \\
\hline $1.0 \cdot 10^{9}$ & $\mathrm{~cm}^{3} \mathrm{~mol}^{-1} \mathrm{yr}^{-1}$ \\
\hline $7.89 \cdot 10^{2}$ & $\mathrm{yr}^{-1}$ \\
\hline $1.0 \cdot 10^{-3}$ & $\mathrm{yr}^{-1}$ \\
\hline 1.6 & $\mathrm{yr}^{-1}$ \\
\hline 1.0 & $\mathrm{yr}^{-1}$ \\
\hline 1.8 & $\mathrm{yr}^{-1}$ \\
\hline 1.0 & $\mathrm{yr}^{-1}$ \\
\hline 400 & $\mathrm{yr}^{-1}$ \\
\hline 1.0 & $\mathrm{yr}^{-1}$ \\
\hline 100 & $\mathrm{yr}^{-1}$ \\
\hline 1.0 & $\mathrm{yr}^{-1}$ \\
\hline $8.470 \cdot 10^{-7}$ & $\mathrm{M}$ \\
\hline $4.300 \cdot 10^{-10}$ & M \\
\hline $1.602 \cdot 10^{-7}$ & M \\
\hline $1.318 \cdot 10^{-9}$ & M \\
\hline
\end{tabular}

Van Cappellen and Wang (1996) Van Cappellen and Wang (1996) Van Cappellen and Wang (1996) Van Cappellen and Wang (1996) Van Cappellen and Wang (1996) Van Cappellen and Wang (1996) LaRowe et al. (2017)

LaRowe et al. (2017)

This study

Thullner et al. (2009)

Van Cappellen and Wang (1996)

Thullner et al. (2009)

Thullner et al. (2009)

Dale et al. (2006)

Van Cappellen and Wang (1996)

Thullner et al. (2009)

Thullner et al. (2009)

Van Cappellen and Wang (1996)

Van Cappellen and Wang (1996)

Thullner et al. (2009)

Thullner et al. (2009)

Van Cappellen and Wang (1996)

Van Cappellen and Wang (1996)

Van Cappellen and Wang (1996)

Van Cappellen and Wang (1996)

Van Cappellen and Wang (1996)

Van Cappellen and Wang (1996)

Van Cappellen and Wang (1996)

Van Cappellen and Wang (1996)

Van Cappellen and Wang (1996)

Van Cappellen and Wang (1996)

Wallmann et al. (2006)

Van Cappellen and Wang (1996)

Pauss et al. (1990)

Van Cappellen and Wang (1996)

Van Cappellen and Wang (1996)

Van Cappellen and Wang (1996)

Van Cappellen and Wang (1996)

Van Cappellen and Wang (1996)

Van Cappellen and Wang (1996)

Van Cappellen and Wang (1996)

Van Cappellen and Wang (1996)

Van Cappellen and Wang (1996)

Millero (1995)

Millero (1995)

Millero (1995)

Millero (1995) 


\begin{tabular}{llll}
{$\left[\mathrm{CH}_{4}\right]^{*}$} & 5.46 & $\mathrm{mM}$ & Dale et al. (2008a) \\
{$\left[\mathrm{PO}_{4}{ }^{3-}\right]^{*}$} & 10 & $\mu \mathrm{M}$ & This study \\
$K_{s \mathrm{MnCO}_{3}}$ & $3.2 \cdot 10^{-9}$ & $\mathrm{M}^{2}$ & Van Cappellen and Wang (1996) \\
$K_{s \mathrm{FeCO}_{3}}$ & $4.0 \cdot 10^{-9}$ & $\mathrm{M}^{2}$ & Van Cappellen and Wang (1996) \\
$K_{s \mathrm{FeS}}$ & $8.2 \cdot 10^{-4}$ & $\mathrm{M}$ & Van Cappellen and Wang (1996) \\
$K_{s \mathrm{CaCO}_{3}}$ & $3.8 \cdot 10^{-7}$ & $\mathrm{M}^{2}$ & Mucci (1983); Millero (1995) \\
\hline$\mu_{g}$ & 18.3 & $\mathrm{yr}^{-1}$ & Dale et al. (2006, 2008b) \\
$\mu_{d}$ & 0.1 & $\mathrm{yr}^{-1}$ & Dale et al. (2006, 2008b) \\
$\gamma$ & 2.48 & - & Dale et al. (2006, 2008b) \\
$\chi$ & 8.0 & - & Dale et al. (2006, 2008b) \\
$\Delta G_{B Q}$ & 20 & $\mathrm{~kJ} / \mathrm{mol} \mathrm{e}^{-}$ & Dale et al. (2006, 2008b) \\
$\Delta G_{r}^{0}$ & -29.92 & $\mathrm{~kJ} / \mathrm{mol} \mathrm{e}^{-}$ & Regnier et al. (2011) \\
$K_{m}^{\mathrm{CH}}$ & $1.5 \cdot 10^{-3}$ & $\mathrm{M}$ & Dale et al. (2006, 2008b) \\
$K_{m}^{\mathrm{SO}_{4}{ }^{2-}}$ & $1.0 \cdot 10^{-3}$ & $\mathrm{M}$ & Dale et al. (2006, 2008b) \\
\hline
\end{tabular}

Table S7. Generic model upper boundary conditions. The boundary condition for biomass is applied only in the bioenergetic formulation of AOM

\begin{tabular}{|c|c|c|c|}
\hline Species & Value@ SWI & Units & Reference \\
\hline POC & 1.04 & $\%$ & This study \\
\hline $\mathrm{O}_{2}$ & 306.98 & $\mu \mathrm{M}$ & Garcia et al. (2010a) \\
\hline $\mathrm{NO}_{3}^{-}$ & 11.51 & $\mu \mathrm{M}$ & Garcia et al. (2010b) \\
\hline $\mathrm{MnO}_{2}$ & 2.18 & $\mu \mathrm{mol} \mathrm{cm}^{-2} \mathrm{yr}^{-1}$ & Dale et al. (2015); Glasby (2006) \\
\hline $\mathrm{Fe}(\mathrm{OH})_{3}$ & 7.09 & $\mu \mathrm{mol} \mathrm{cm}^{-2} \mathrm{yr}^{-1}$ & Dale et al. (2015); Glasby (2006) \\
\hline $\mathrm{SO}_{4}^{2-}$ & 28.0 & $\mathrm{mM}$ & Thullner et al. (2009) \\
\hline $\mathrm{CH}_{4}$ & 0.0 & $\mathrm{mM}$ & This study \\
\hline $\mathrm{NH}_{4}^{+}$ & 0.0 & $\mathrm{mM}$ & This study \\
\hline $\mathrm{PO}_{4}^{3-}$ & 1.0 & $\mu \mathrm{M}$ & Sales de Freitas (2018) \\
\hline $\mathrm{Mn}^{2+}$ & 1.0 & $\mu \mathrm{M}$ & Sales de Freitas (2018) \\
\hline $\mathrm{Fe}^{2+}$ & 1.0 & $\mathrm{pM}$ & Sales de Freitas (2018) \\
\hline $\mathrm{H}_{2} \mathrm{~S}$ & 0.835 & $\mathrm{nM}$ & Sales de Freitas (2018) \\
\hline $\mathrm{HS}^{-}$ & 9.17 & $\mathrm{nM}$ & Sales de Freitas (2018) \\
\hline $\mathrm{CH}_{4}(\mathrm{~g})$ & 0.0 & $\mathrm{nM}$ & This study \\
\hline $\mathrm{H}_{2} \mathrm{CO}_{3}$ & 39 & $\mu \mathrm{M}$ & Sales de Freitas (2018) \\
\hline $\mathrm{HCO}_{3}{ }^{-}$ & 2150 & $\mu \mathrm{M}$ & Sales de Freitas (2018) \\
\hline $\mathrm{CO}_{3}^{2-}$ & 15.3 & $\mu \mathrm{M}$ & Sales de Freitas (2018) \\
\hline $\mathrm{B}(\mathrm{OH})_{3}$ & 363.6 & $\mu \mathrm{M}$ & Sales de Freitas (2018) \\
\hline $\mathrm{B}(\mathrm{OH})_{4}^{-}$ & 61.4 & $\mu \mathrm{M}$ & Sales de Freitas (2018) \\
\hline $\mathrm{H}^{+}$ & 6.31 & $\mu \mathrm{M}$ & Sales de Freitas (2018) \\
\hline $\mathrm{CaCO}_{3}$ & 23 & $\mu \mathrm{mol} \mathrm{cm}{ }^{-2} \mathrm{yr}^{-1}$ & Sales de Freitas (2018) \\
\hline $\mathrm{Ca}^{2+}$ & 9.7 & $\mathrm{mM}$ & Sales de Freitas (2018) \\
\hline $\mathrm{NH}_{4}^{+}(\mathrm{ads})$ & 0 & $\mathrm{mM}$ & Sales de Freitas (2018) \\
\hline $\mathrm{PO}_{4}{ }^{3-}$ (ads) & 0 & $\mathrm{mM}$ & Sales de Freitas (2018) \\
\hline $\mathrm{FeS}$ & 0 & $\mu \mathrm{mol} \mathrm{cm}^{-2} \mathrm{yr}^{-1}$ & Sales de Freitas (2018) \\
\hline $\mathrm{FeCO}_{3}$ & 0 & $\mathrm{mM}$ & Sales de Freitas (2018) \\
\hline
\end{tabular}




\begin{tabular}{llll}
$\mathrm{S}^{0}$ & 0 & $\mathrm{mM}$ & Sales de Freitas (2018) \\
$\mathrm{FeS}_{2}$ & 0 & $\mu \mathrm{mol} \mathrm{cm}^{-2} \mathrm{yr}^{-1}$ & Sales de Freitas (2018) \\
$\mathrm{Fe}^{2+}(\mathrm{ads})$ & 0 & $\mathrm{mM}$ & Sales de Freitas (2018) \\
$\mathrm{PO}_{4}\left(\mathrm{ads}_{\mathrm{Fe}(\mathrm{OH}) 3}\right)$ & 0 & $\mathrm{Mm}$ & Sales de Freitas (2018) \\
\hline $\mathrm{B}$ & $1.0 \cdot 10^{8}, 3.158 \cdot 10^{7}$ & $\mathrm{~mol} \mathrm{~cm}^{-3}$, cells cm$^{-3}$ & This study \\
\hline
\end{tabular}

Table S8. AOM Damköhler number for $\omega=0.123 \mathrm{~cm} \mathrm{yr}^{-1}$ and $\omega=1.5 \mathrm{~cm} \mathrm{yr}^{-1}$. The reported values are the average of the maximum and minimum resulting from simulations with different bottom methane concentration. Missing values are because simulations were not run with the corresponding pair of parameters.

\begin{tabular}{cc|ccccccc}
\hline & & \multicolumn{7}{c}{$v_{u p}\left[\mathrm{~cm} \mathrm{yr}^{-1}\right]$} \\
\cline { 3 - 8 } & & 0 & 0.3 & 0.5 & 1 & 3 & 7 & 10 \\
\hline \multirow{2}{*}[\mathrm{cm}\mathrm{yr}^{-1}]{} & 0.123 & 1206 & 1124 & 683 & 327 & 120 & 52 & 32 \\
& & 1521 & 1473 & 772 & 409 & 139 & 57 & 42 \\
\cline { 2 - 8 } & 1.5 & 470 & - & - & 1408 & - & - & - \\
\hline
\end{tabular}

Table S9. Name, location, water depth and linear sedimentation rate for cores used to inter- and extra-polate the sedimentation rates to the whole Laptev sea area via simple 3D kriging.

\begin{tabular}{llllll}
\hline Core name & Lon $\left({ }^{\circ}\right)$ & Lat $\left({ }^{\circ}\right)$ & Water depth $(\mathrm{m})$ & $\omega(\mathrm{cm} / \mathrm{yr})$ & Reference \\
\hline KD9502-14 & 133.117 & 76.192 & 46 & 0.003 & Bauch et al. (2001) \\
PM9462-4 & 136.005 & 74.503 & 27 & 0.05 & Bauch et al. (2001) \\
PM9499-2 & 115.545 & 75.501 & 48 & 0.004 & Bauch et al. (2001) \\
PM9402-3 & 115.249 & 75.491 & 47 & 0.16 & Strobl et al. (1998) \\
PM9417-4 & 130.014 & 75.503 & 51 & 0.08 & Strobl et al. (1998) \\
PM9442-3 & 126.003 & 74.501 & 40 & 0.13 & Strobl et al. (1998) \\
PM9462-1 & 136.004 & 74.502 & 27 & 0.12 & Strobl et al. (1998) \\
PM9463-8 & 126.582 & 74.504 & 36 & 0.35 & Strobl et al. (1998) \\
PM9481-2 & 134.004 & 73.750 & 17 & 0.17 & Strobl et al. (1998) \\
PM9482-1 & 128.175 & 73.999 & 27 & 0.39 & Strobl et al. (1998) \\
PS51/080-13 & 131.638 & 73.459 & 21 & 0.028 & Bauch et al. (2001) \\
PS51/092-13 & 130.136 & 74.594 & 32 & 0.07 & Bauch et al. (2001) \\
PS51/092-12 & 130.138 & 74.593 & 32 & 0.041 & Bauch et al. (2001) \\
PS51/118-2 & 132.237 & 77.892 & 114 & 0.002 & Bauch et al. (2001) \\
PS51/118-3 & 132.199 & 77.892 & 122 & 0.008 & Bauch et al. (2001) \\
PS51/135-4 & 133.243 & 76.165 & 51 & 0.031 & Bauch et al. (2001) \\
PS51/141-2 & 128.641 & 75.227 & 42 & 0.011 & Bauch et al. (2001) \\
PS51/154-11 & 120.610 & 77.276 & 270 & 0.012 & Bauch et al. (2001) \\
PS51/159-10 & 116.032 & 76.767 & 60 & 0.011 & Bauch et al. (2001) \\
PS2458-4 & 133.398 & 78.167 & 983 & 0.027 & Bauch et al. (2001) \\
L13-09-2 & 129.895 & 73.018 & 10 & 0.45 & Han (2014) \\
L13-14-2 & 130.695 & 73.857 & 25 & 0.16 & Han (2014)
\end{tabular}




\begin{tabular}{|c|c|c|c|c|c|}
\hline L13-18-2 & 130.479 & 73.032 & 16 & 0.3 & Han (2014) \\
\hline L13-04-2 & 130.690 & 71.902 & 15 & 0.2 & Han (2014) \\
\hline $\mathrm{C}-37$ & 130.367 & 71.617 & 10 & 0.024 & Stein and Fahl (2000) \\
\hline C-4 & 131.000 & 73.167 & 26 & 0.17 & Stein and Fahl (2000) \\
\hline $\mathrm{C}-7$ & 129.983 & 74.883 & 37 & 0.159 & Stein and Fahl (2000) \\
\hline C-8 & 130.500 & 75.400 & 48 & 0.015 & Stein and Fahl (2000) \\
\hline C-11 & 130.083 & 76.867 & 66 & 0.011 & Stein and Fahl (2000) \\
\hline PS2725-5 & 144.135 & 78.656 & 77 & 0.013 & Stein et al. (2001) \\
\hline PS2778-2 & 113.065 & 77.978 & 341 & 0.038 & Stein et al. (2001) \\
\hline PS2476-4 & 118.193 & 77.39 & 521 & 0.05 & Stein et al. (2001) \\
\hline PS2742-5 & 103.815 & 80.788 & 1890 & 0.022 & Stein et al. (2001) \\
\hline PS2474-3 & 118.575 & 77.67 & 1494 & 0.02 & Stein et al. (2001) \\
\hline PS2741-1 & 105.395 & 81.105 & 2400 & 0.012 & Stein et al. (2001) \\
\hline PS2471-4 & 119.793 & 79.152 & 3047 & 0.002 & Stein et al. (2001) \\
\hline
\end{tabular}


S6.1 Sensitivity study: model parameters and model boundary conditions. Figures
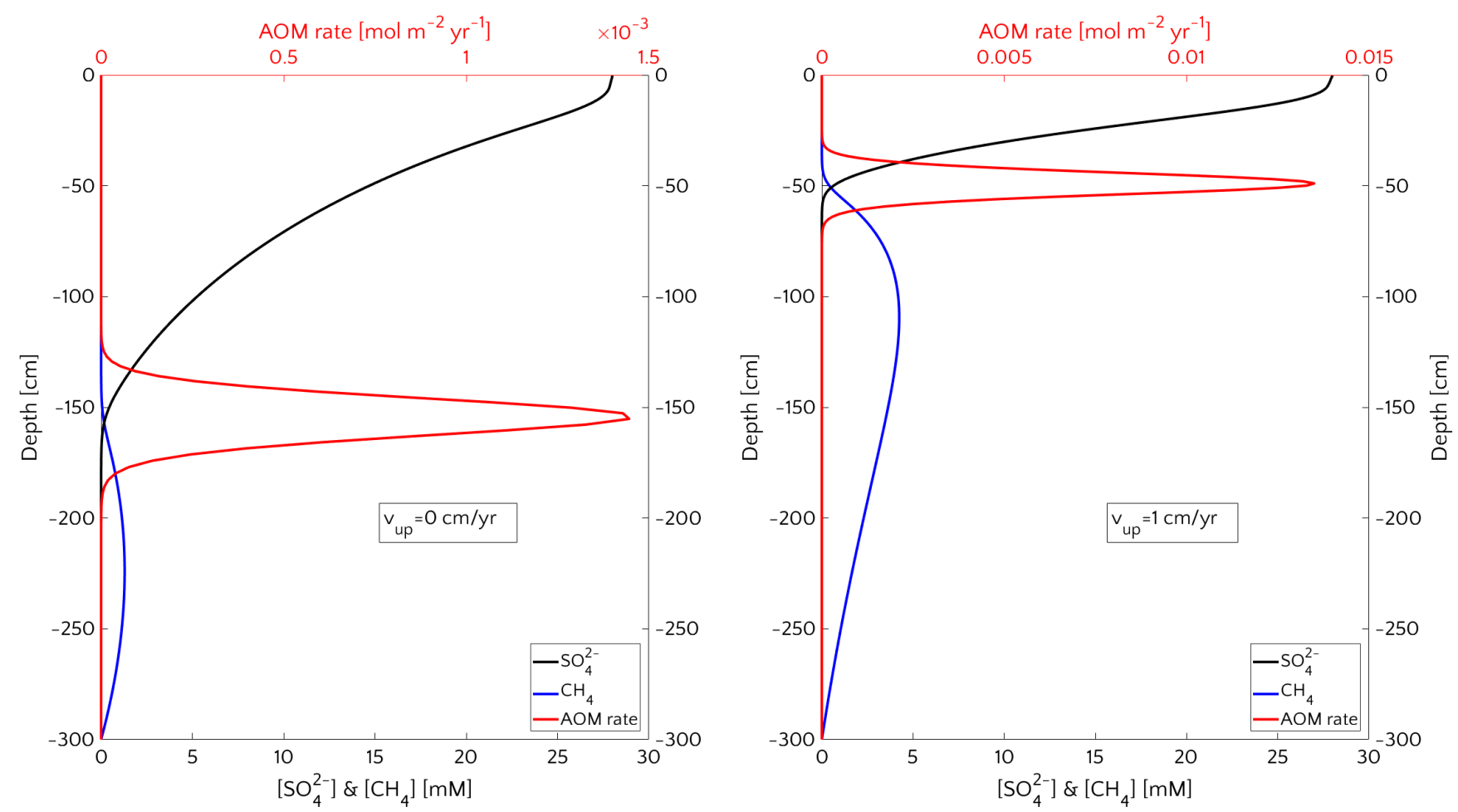

Figure S1. Outcomes for the baseline simulations at steady state. Passive case (left) and active case with $v_{u p}=1 \mathrm{~cm} / \mathrm{yr}$ (right). Typical SMTZ shoaling (from $155.2 \mathrm{~cm}$ to $48.9 \mathrm{~cm}$ ) and squeezing (from $65 \mathrm{~cm}$ to $38 \mathrm{~cm}$ ) from passive to active case. 


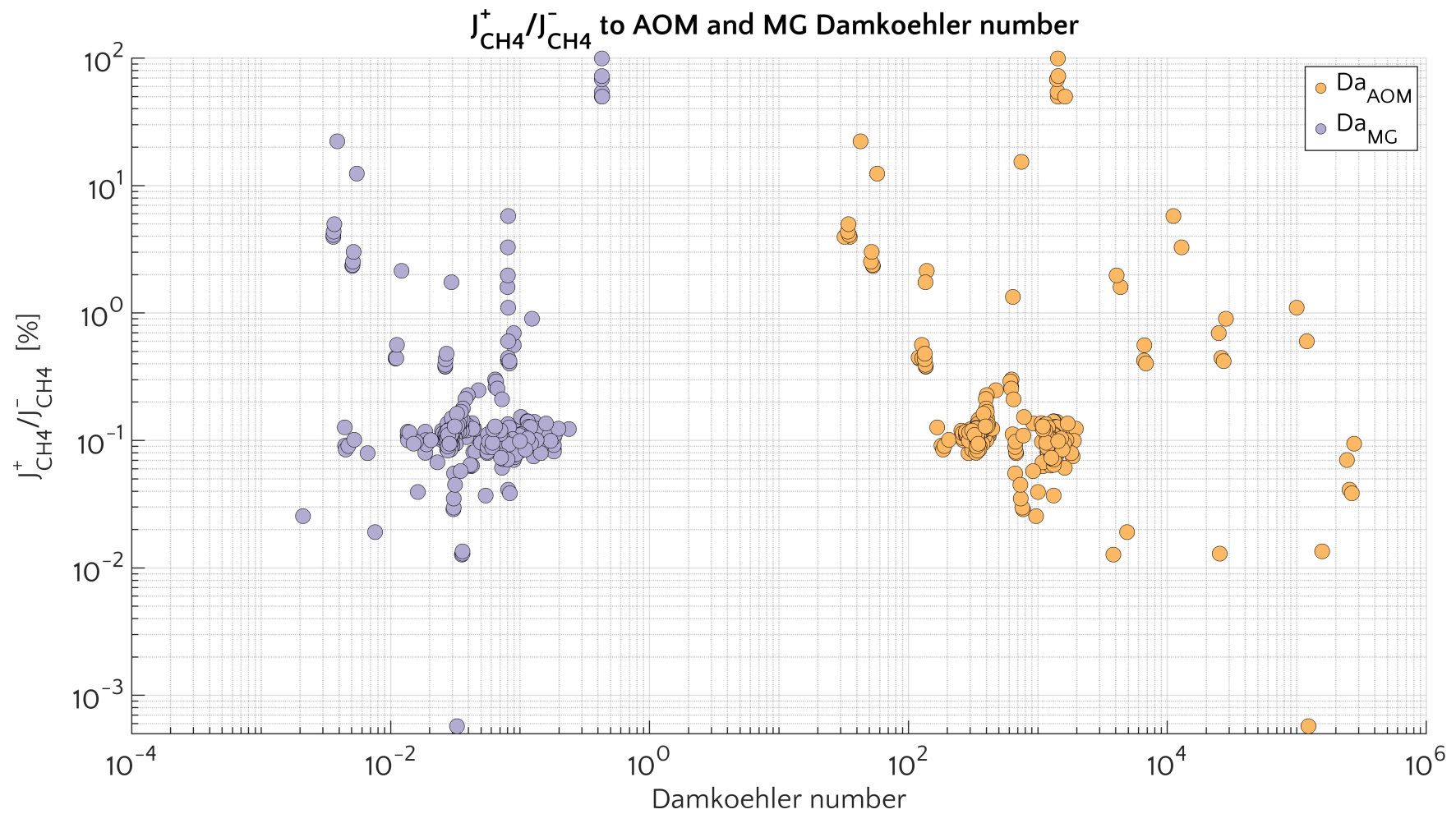

Figure S2. Ratio $J_{C H 4}^{+} / J_{C H 4}^{-}$(in \%) as a function pf the Damköler numbers for AOM (orange) and for methanogenesis (purple) 


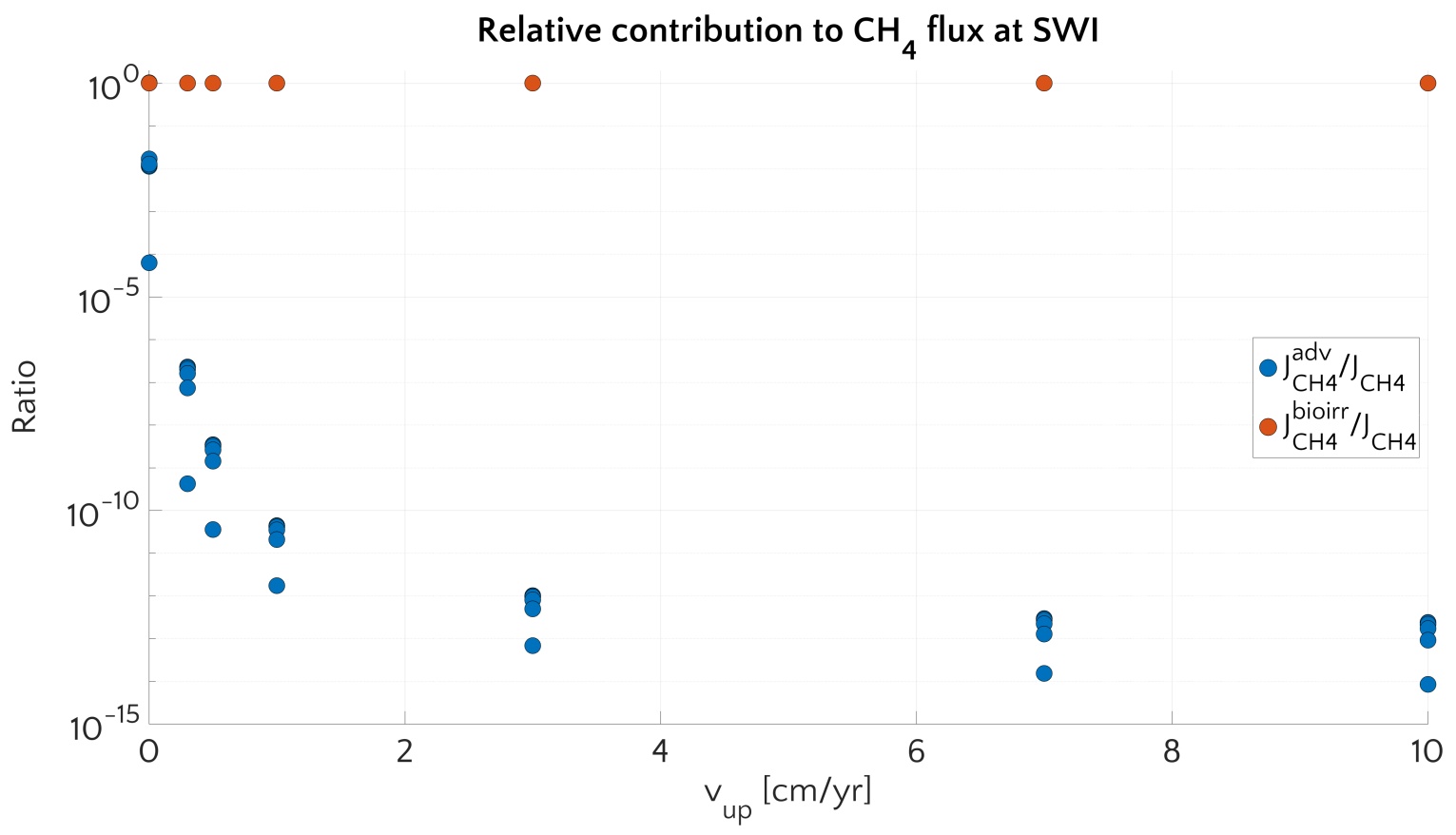

Figure S3. Relative contribution of transport process to the methane flux at the SWI: the advective component (blue) and the bioirrigation component (red). $\omega$ is set to the baseline value of $0.123 \mathrm{~cm} \mathrm{yr}^{-1}$. For each value of $v_{u p}$ and a specific flux component each dot corresponds to a simulation with a different value of bottom $\mathrm{CH}_{4}$ concentration. Diffusive component of the flux is always $<10^{-10}$. 


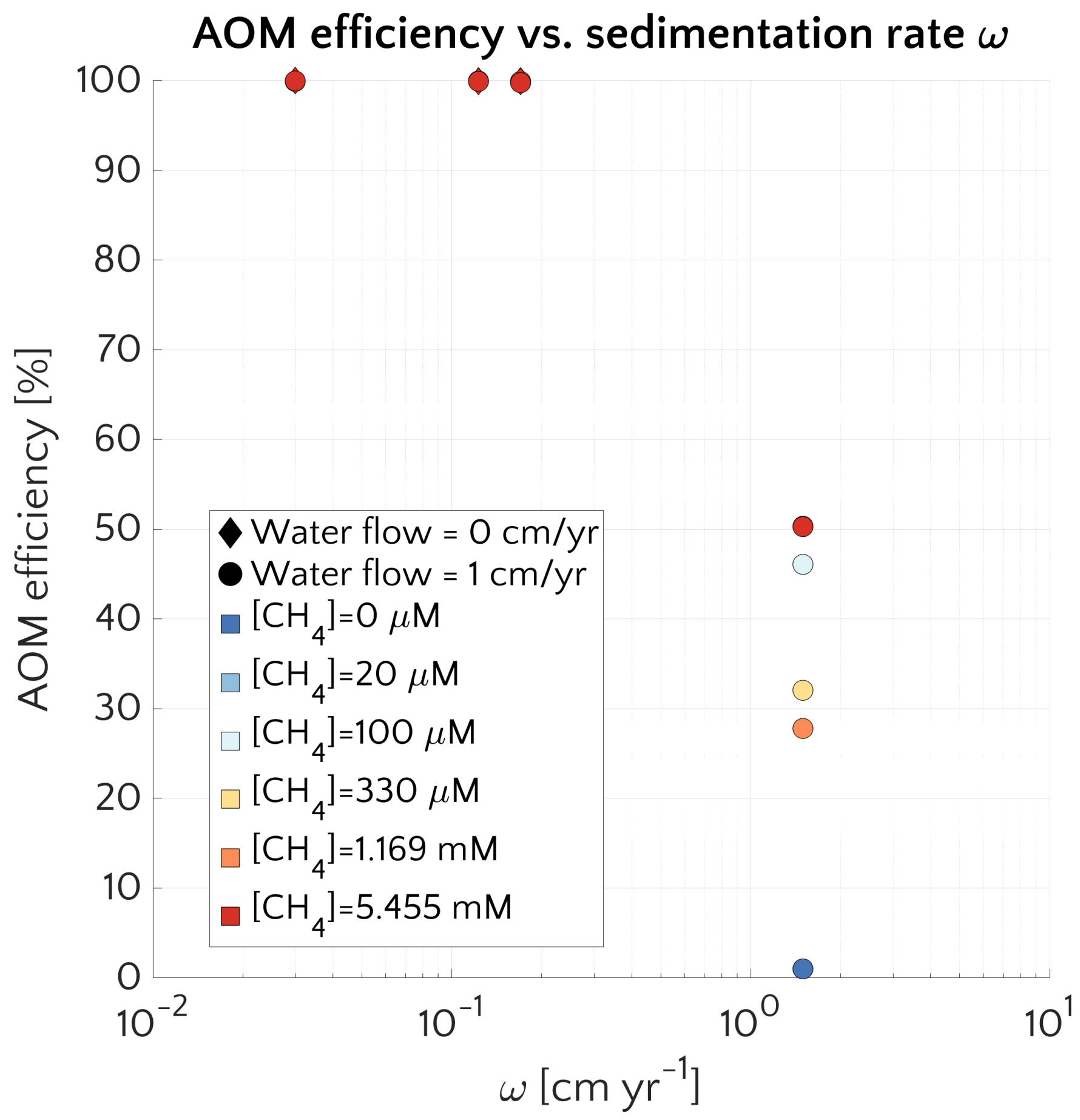

Figure S4. AOM filter efficiency $\eta$ versus $\omega$ for passive cases (diamonds) and active cases (circles). 


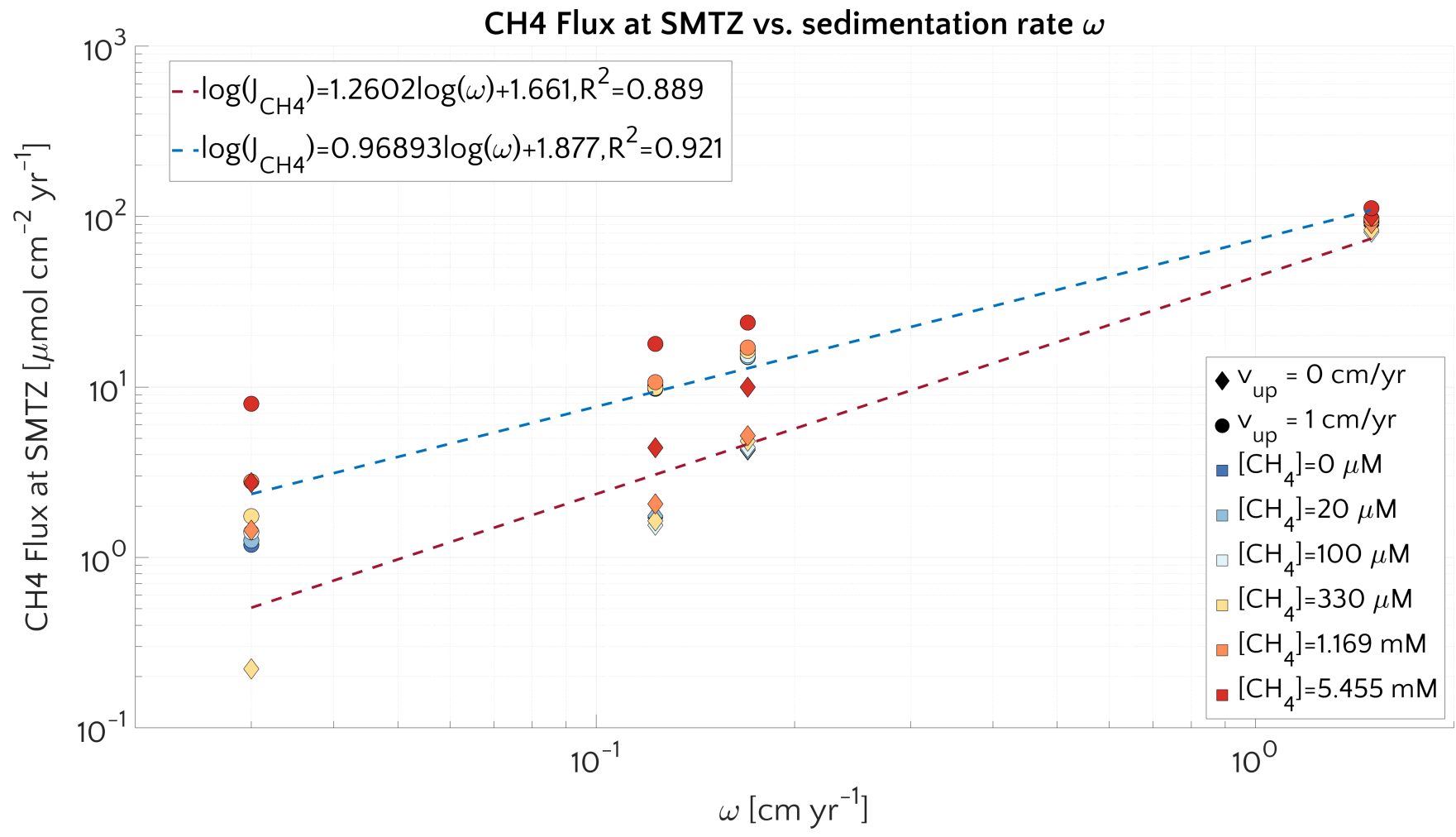

Figure S5. Methane flux at the centre of SMTZ versus $\omega$ for passive (diamonds) and active (circle) cases. Log-log fit is reported. 


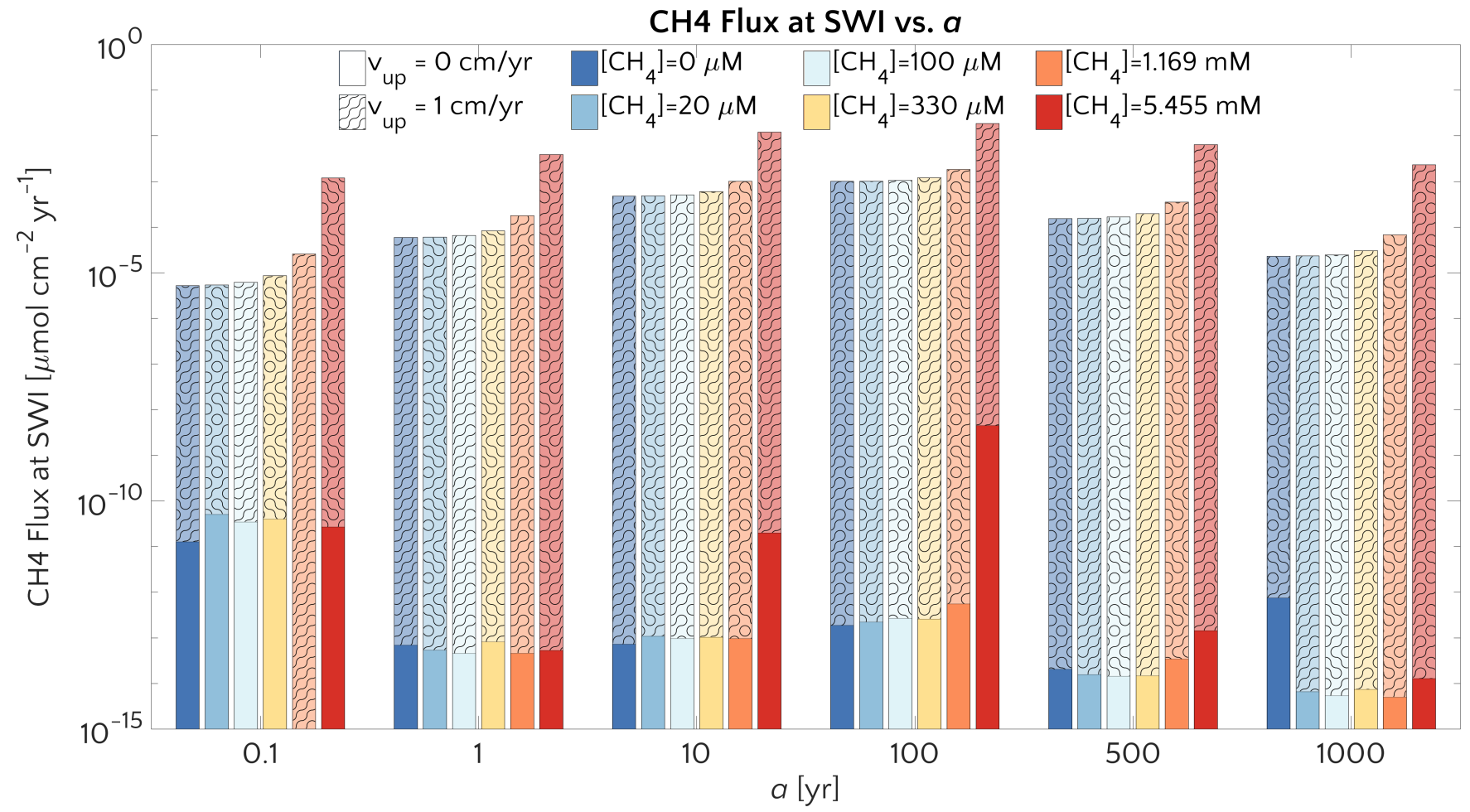

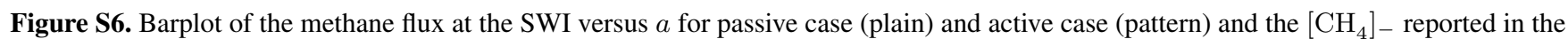
text. 


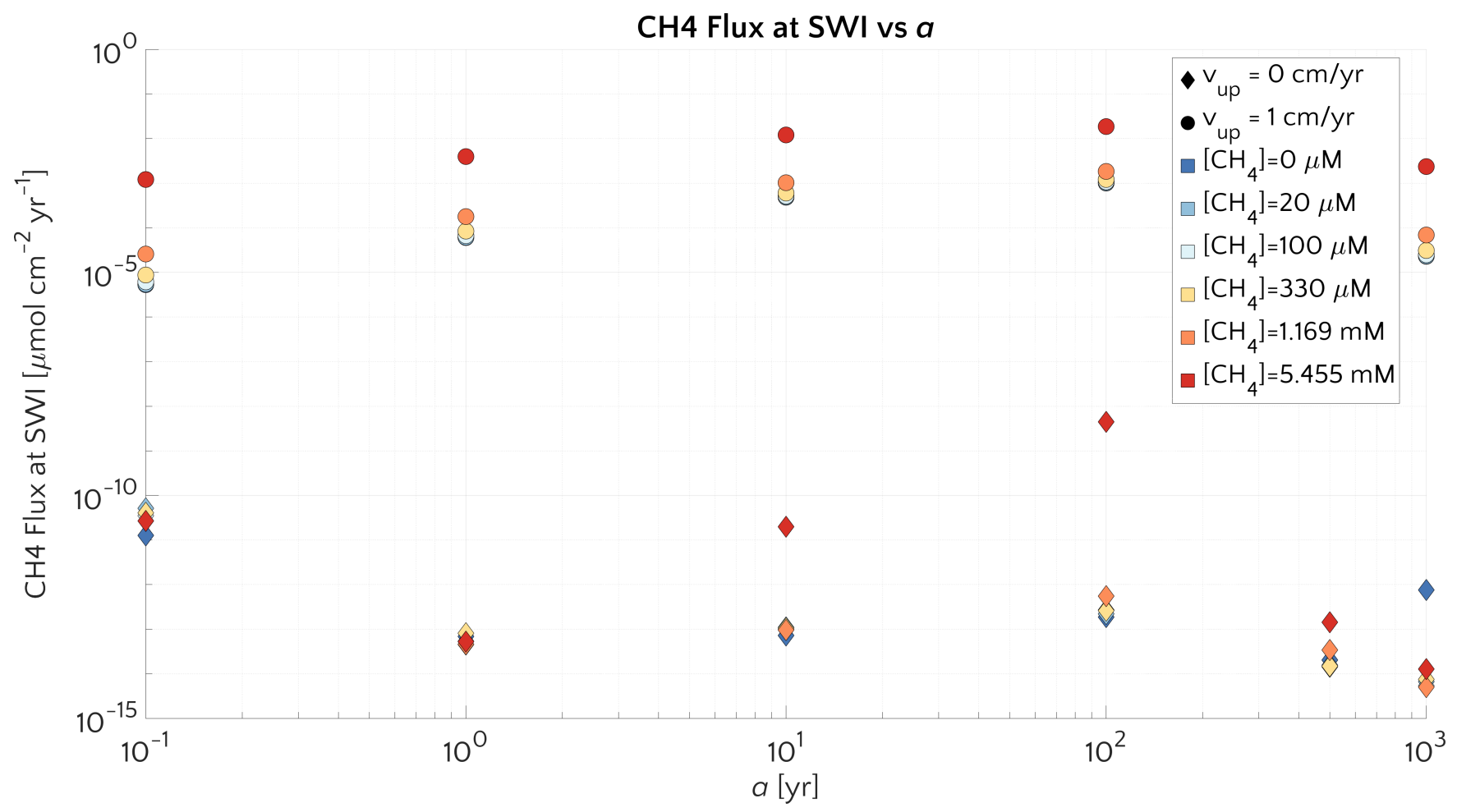

Figure S7. Methane flux at SWI versus $a$ for passive (diamonds) and active (circle) cases. 


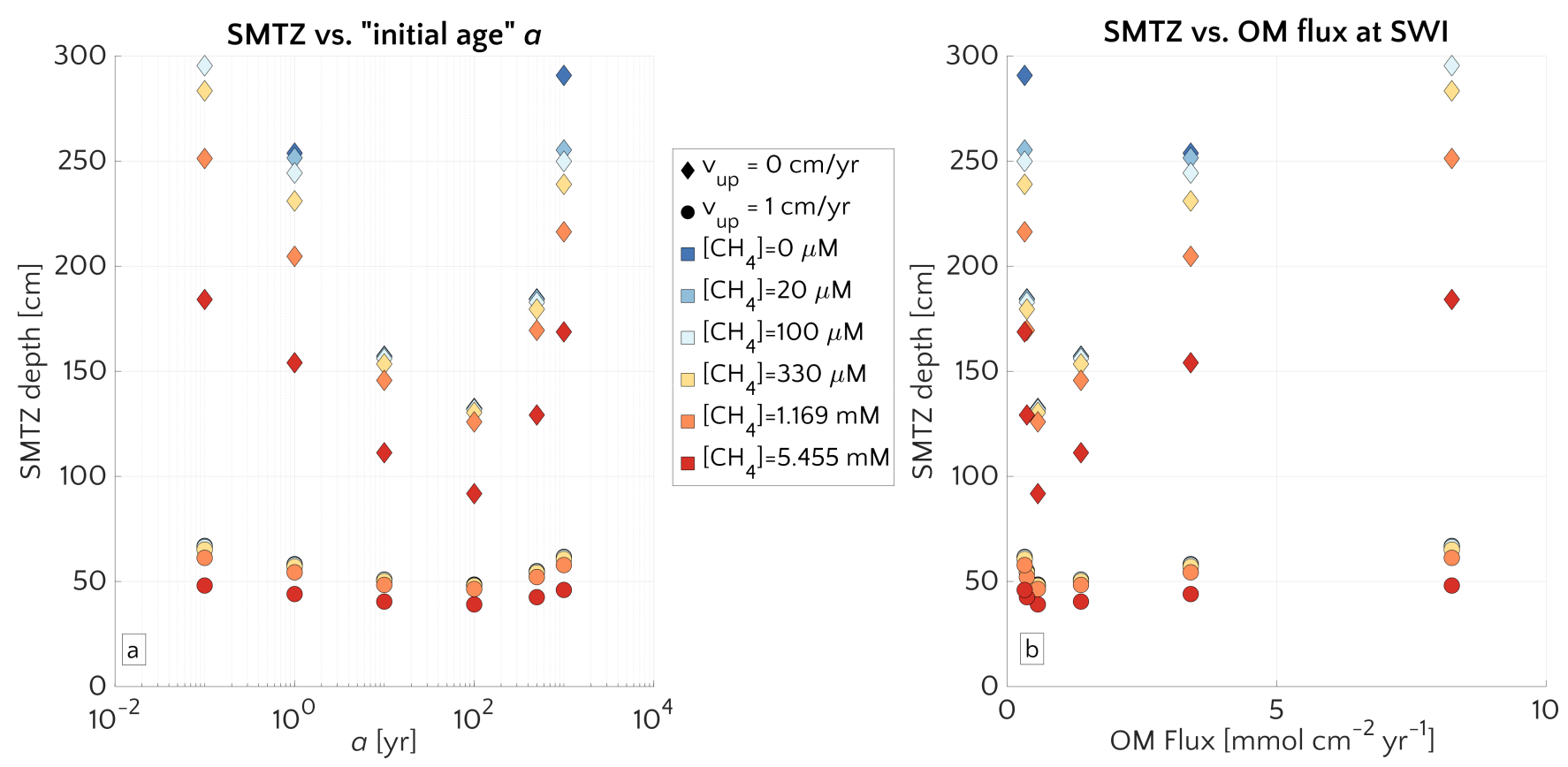

Figure S8. (a) SMTZ depth versus $a$. Passive (diamonds) and active (circle) cases for different $\left[\mathrm{CH}_{4}\right]_{-}$. (b) SMTZ depth versus OM flux entering the system at the SWI. 


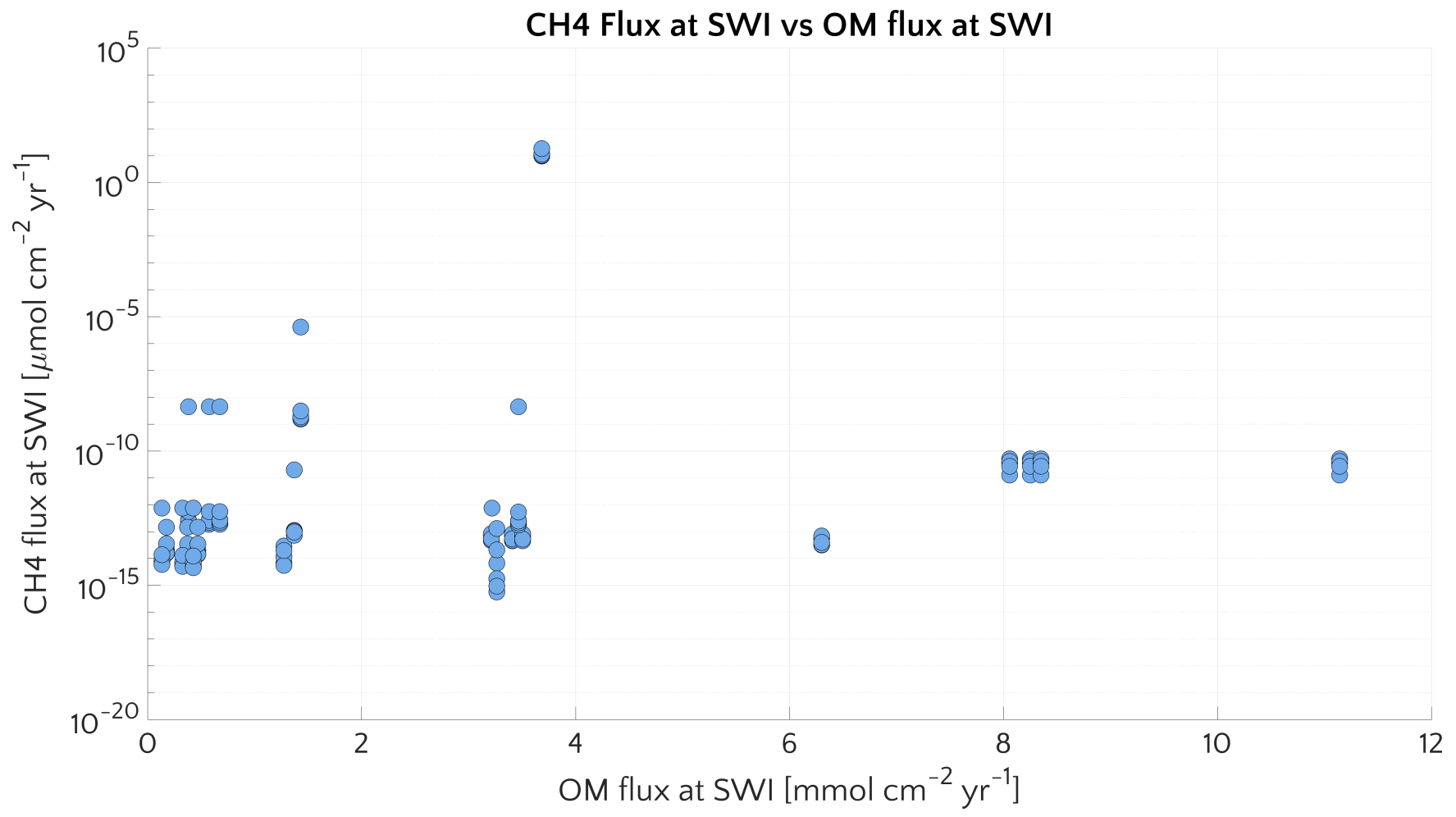

Figure S9. Methane flux at SWI versus OM flux at SWI 


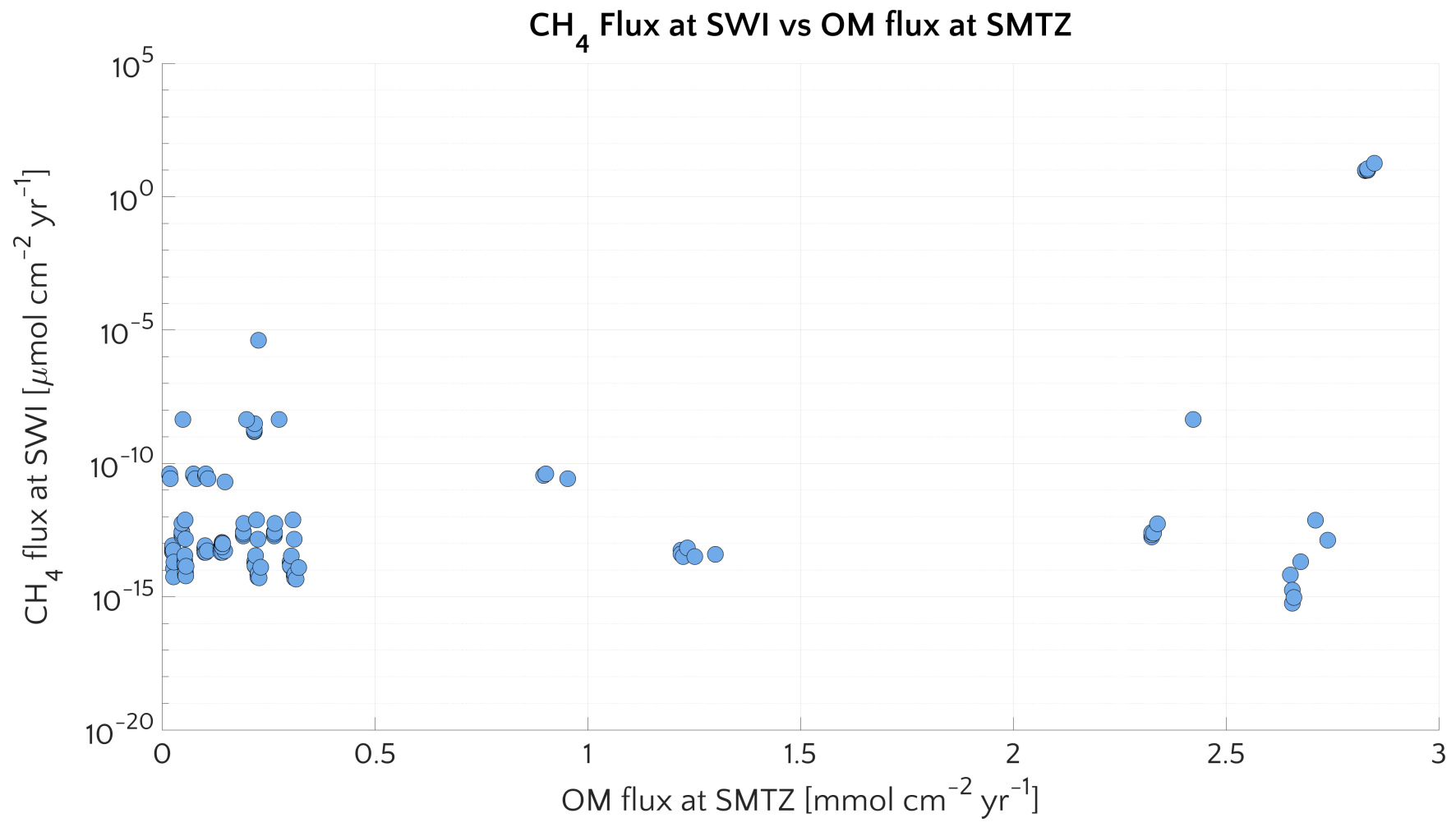

Figure S10. Methane flux at SWI versus OM flux at SMTZ 


\section{$\mathrm{CH}_{4}$ Flux at SWI vs OM content}

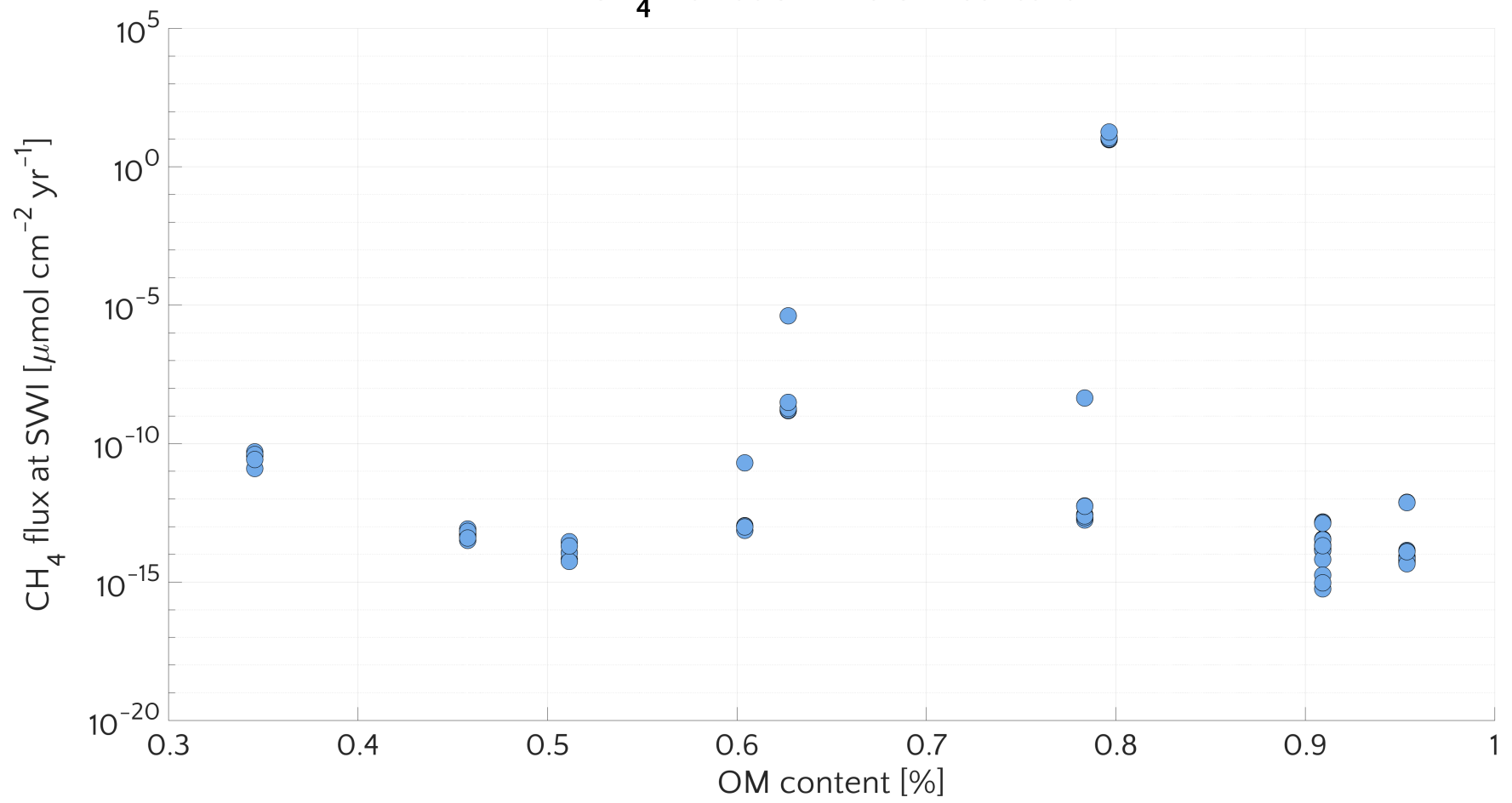

Figure S11. Methane flux at SWI versus OM sediment content 


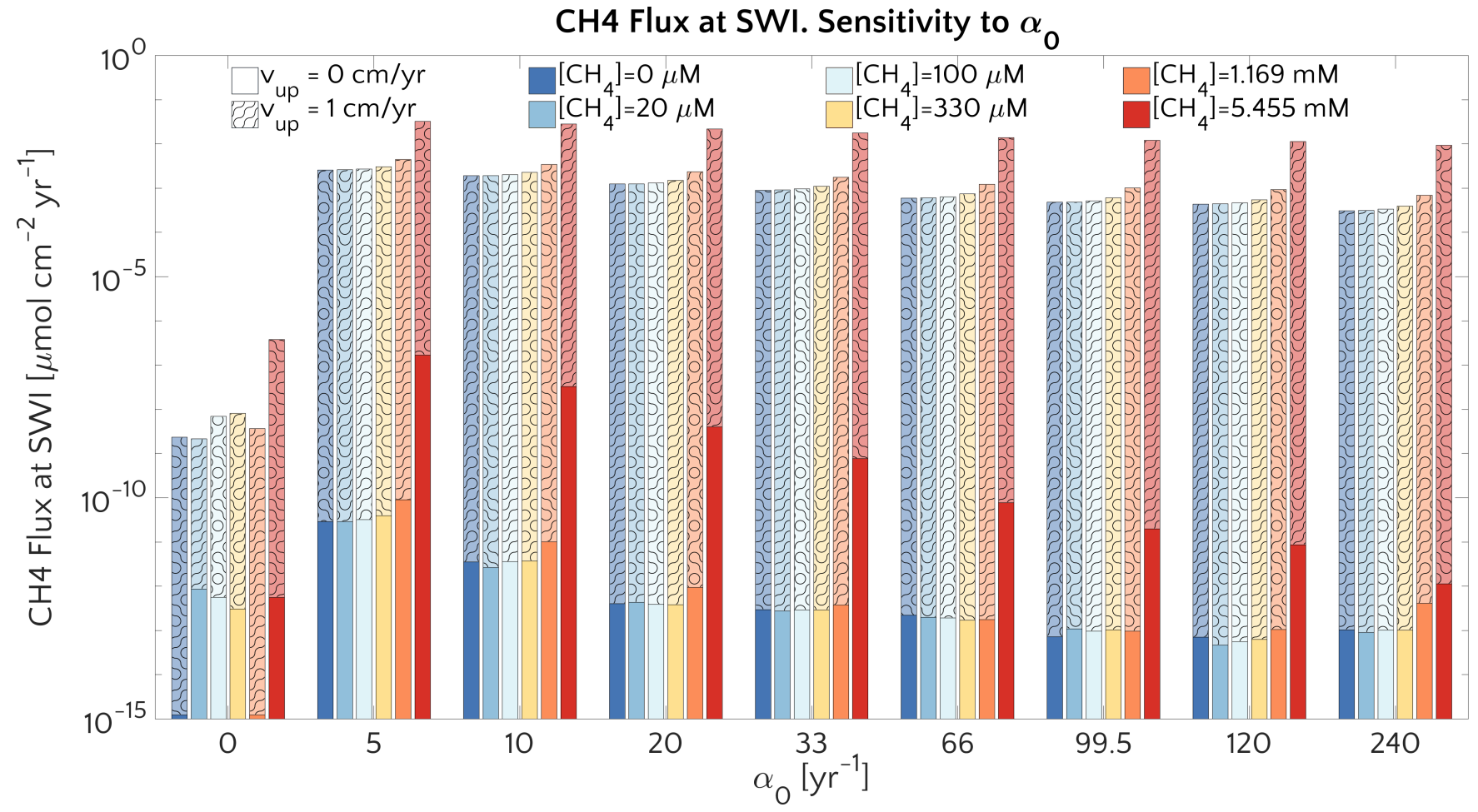

Figure S12. Barplot of the methane flux at the SWI versus $\alpha_{0}$ for passive case (plain style) and active case (pattern style) and the $\left[\mathrm{CH}_{4}\right]_{-}$ analyzed. 

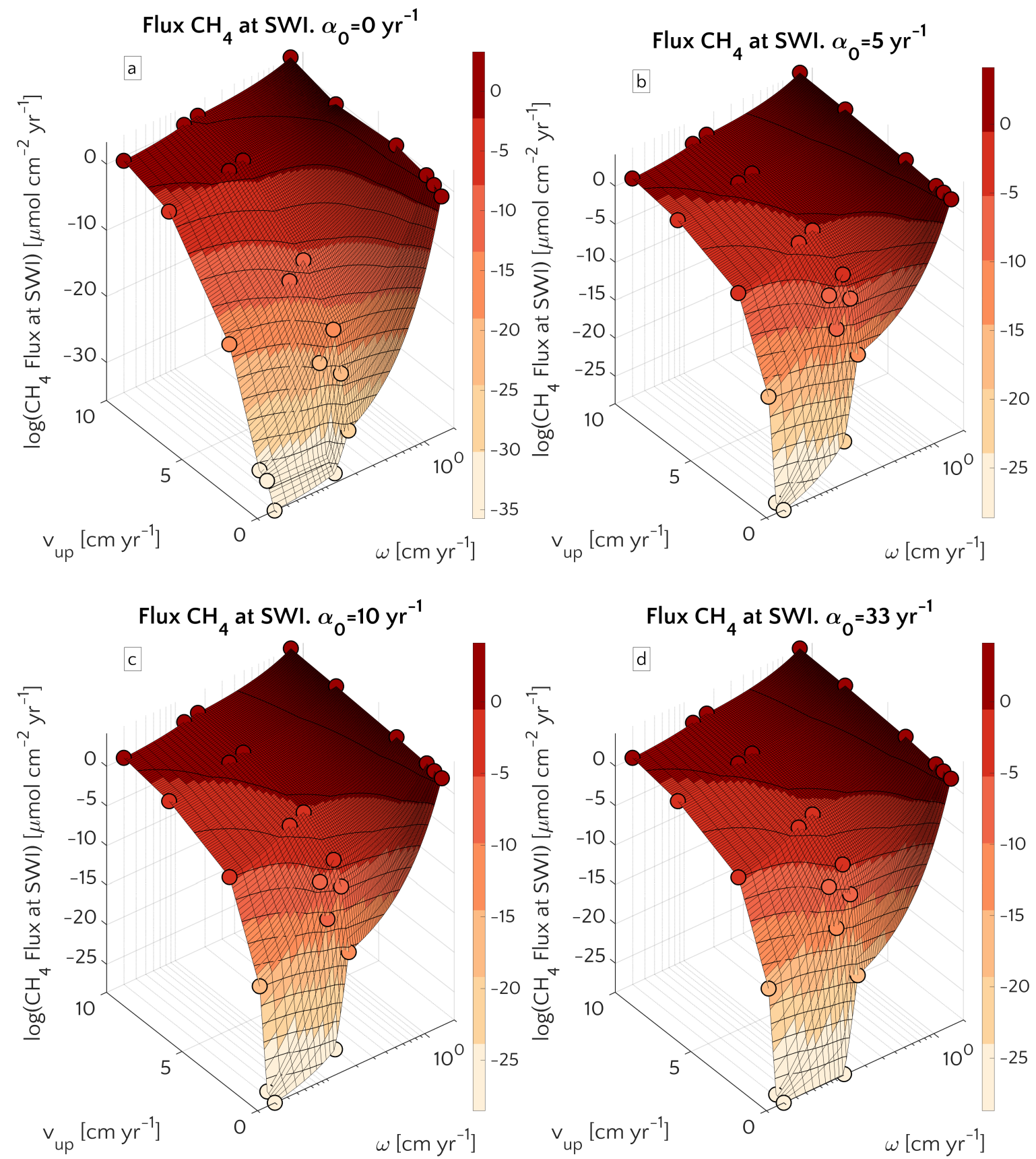

Figure S13. 3D plot of efflux of methane at the SWI as dependent on $v_{u p}$ and $\omega$ for $\alpha_{0}=0 \mathrm{yr}^{-1}(a), \alpha_{0}=5 \mathrm{yr}^{-1}(b), \alpha_{0}=10 \mathrm{yr}^{-1}(c)$ and $\alpha_{0}=33 \mathrm{yr}^{-1}(d)$. Circles represent simulations outcomes. Results for $\alpha_{0} \neq 0 \mathrm{yr}^{-1}$ are almost the same. The lower boundary condition for methane is $\left[\mathrm{CH}_{4}\right]_{-}=1.169 \mathrm{mM}$. 


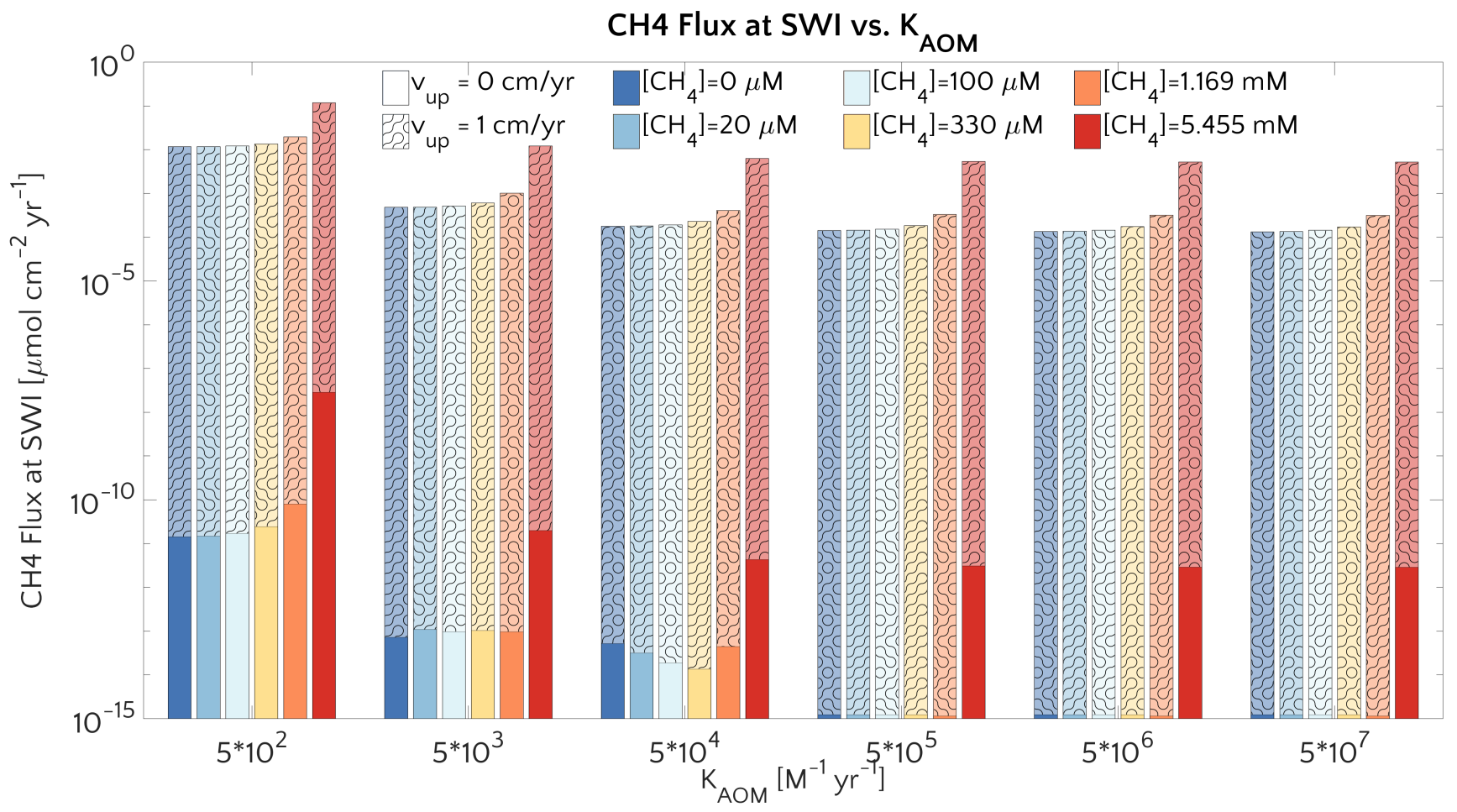

Figure S14. Methane flux at SWI versus AOM reactivity constant $k_{A O M}$ 


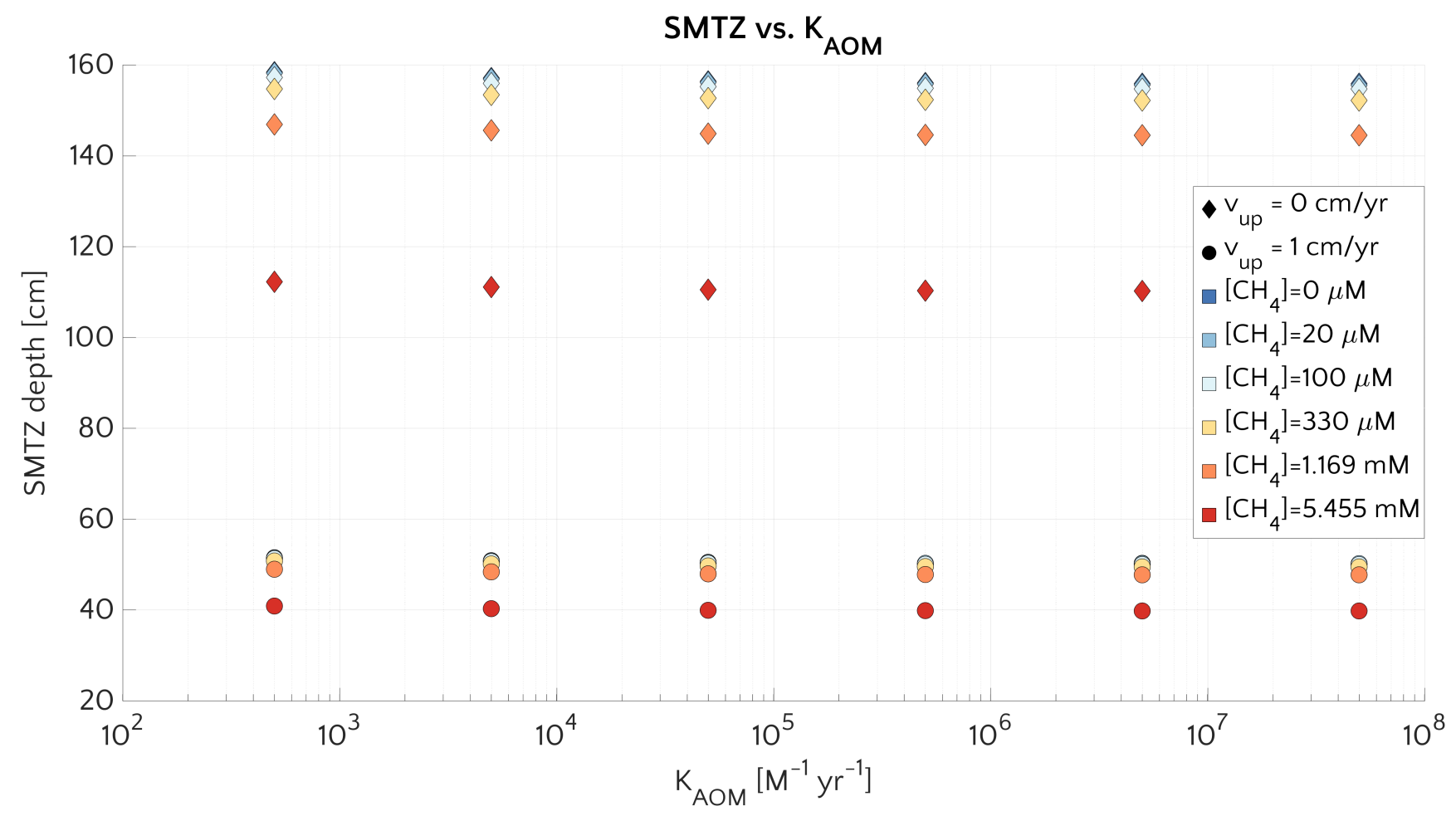

Figure S15. SMTZ depth versus $k_{A O M}$. Passive (diamonds) and active (circle) cases for different $\left[\mathrm{CH}_{4}\right]_{-}$. 

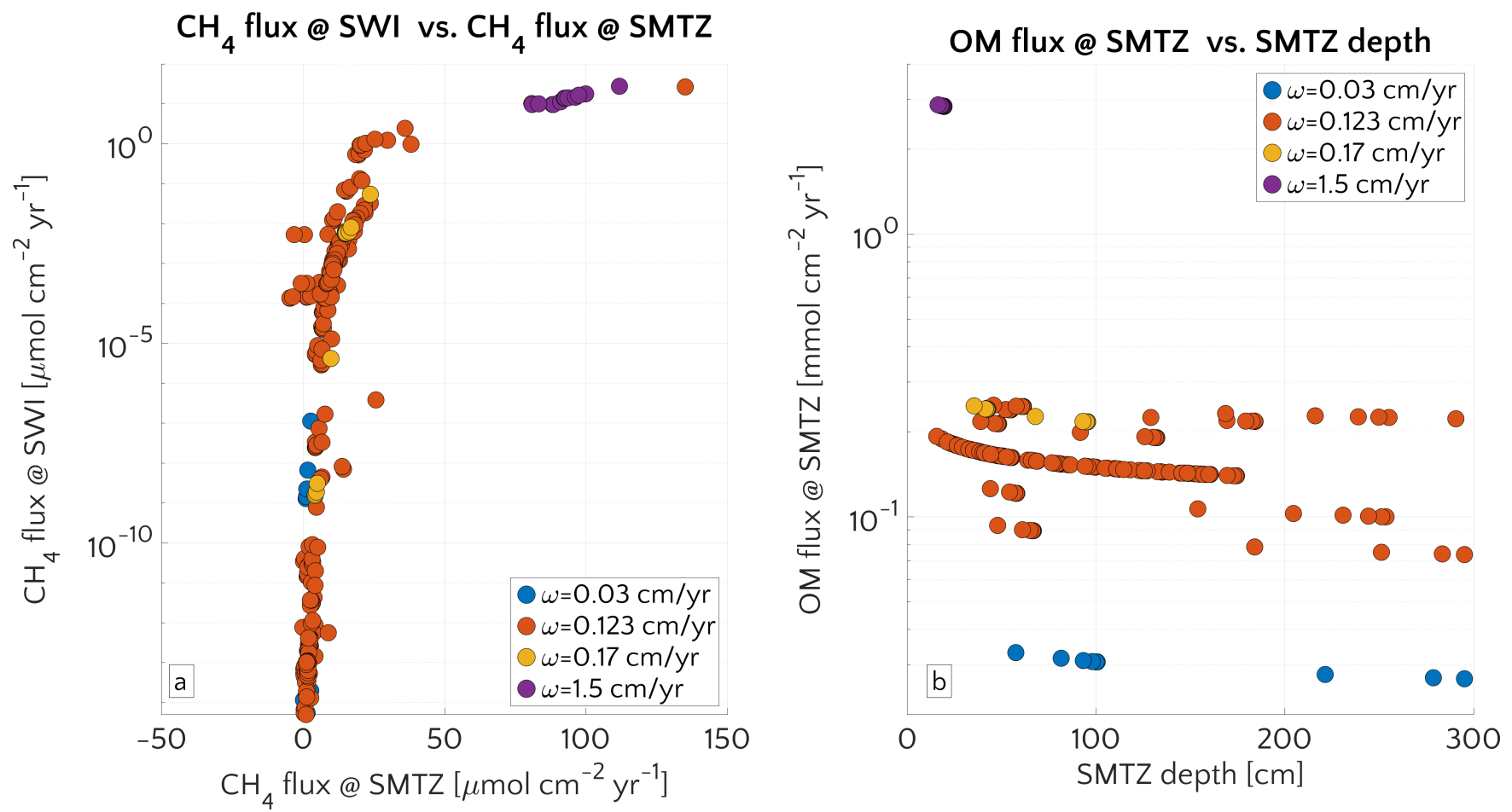

Figure S16. $a$. Flux of methane at SWI versus flux of methane at the SMTZ depth. $b$. OM flux at the SMTZ depth versus SMTZ depth itself. Colours identify simulations with different $\omega$. 

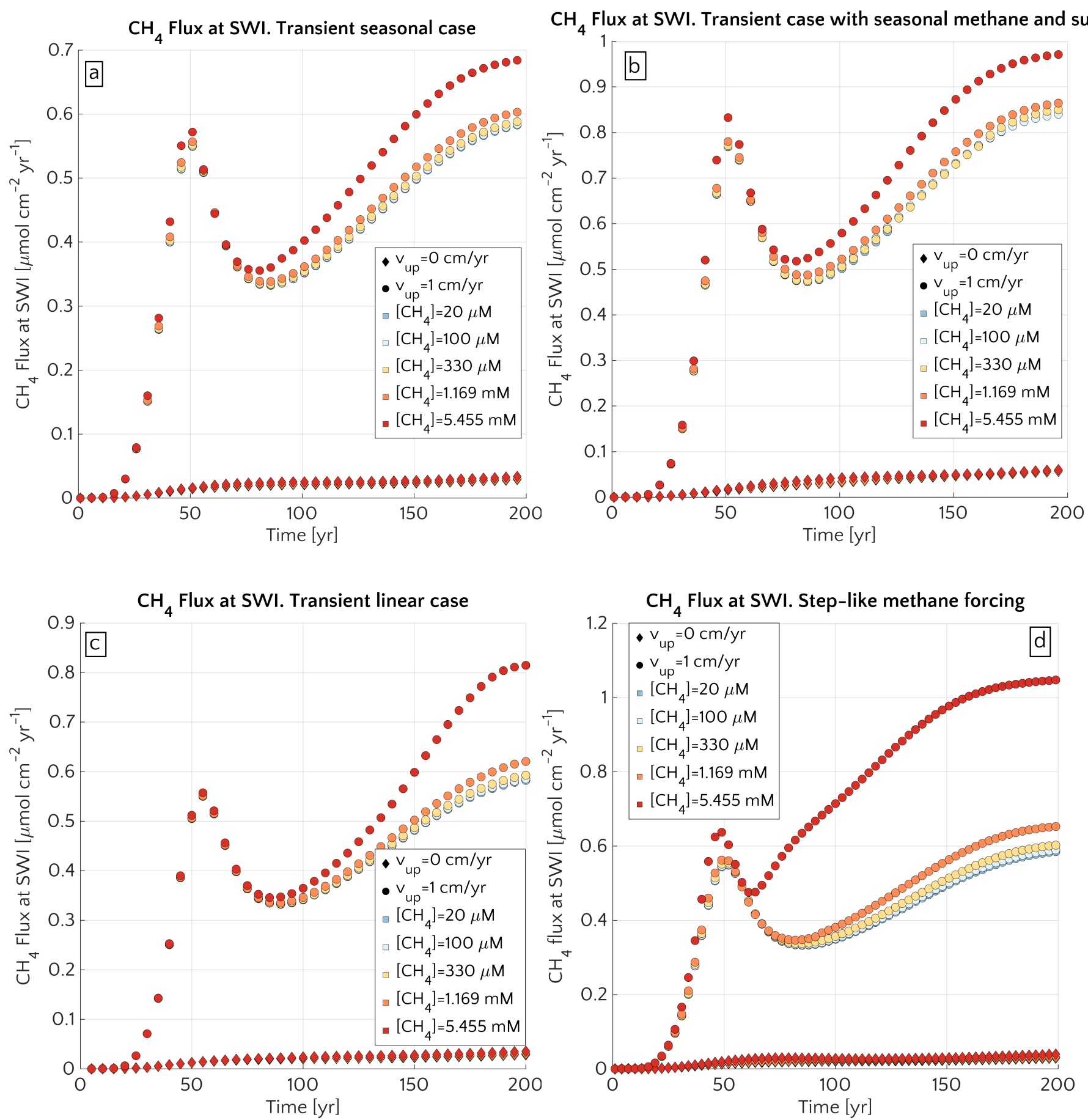

Figure S17. Flux of methane at SWI over time for passive (diamonds) and active (circle) setups and different $\left[\mathrm{CH}_{4}\right]_{-} . a$. Seasonal methane forcing from below. $b$. Seasonal methane forcing from below and seasonal sulfate forcing from above. $c$. Linear methane forcing from below. $d$. Step-like methane forcing from below. 


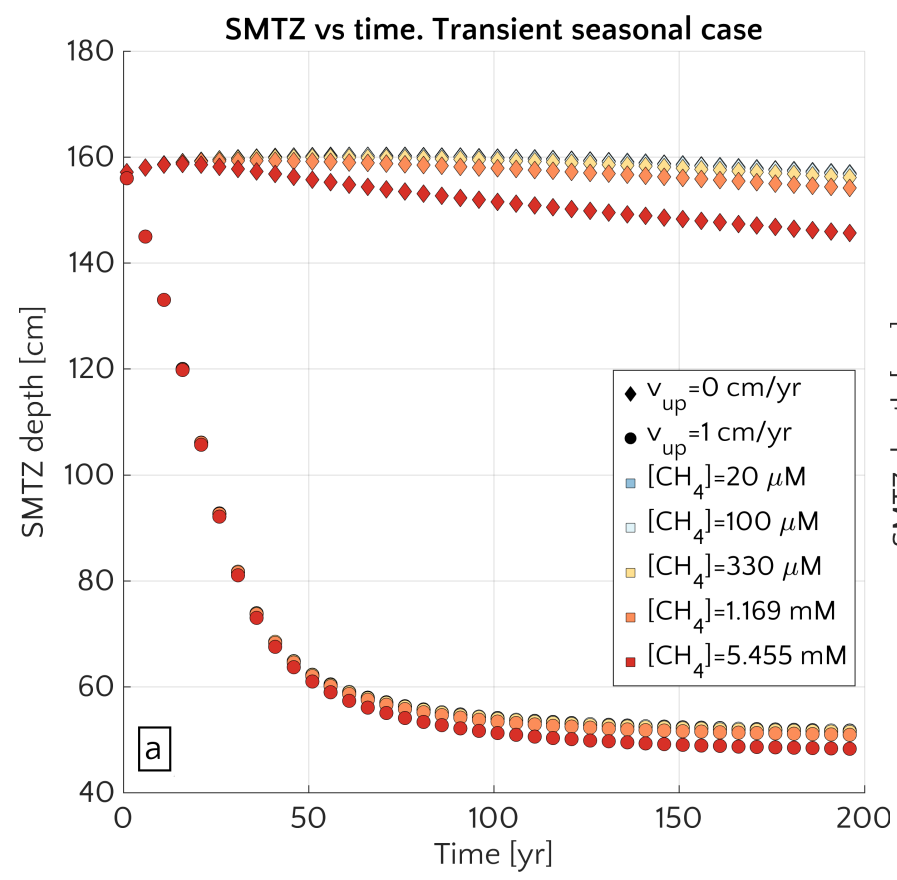

SMTZ vs time. Transient case with seasonal methane and sulfate
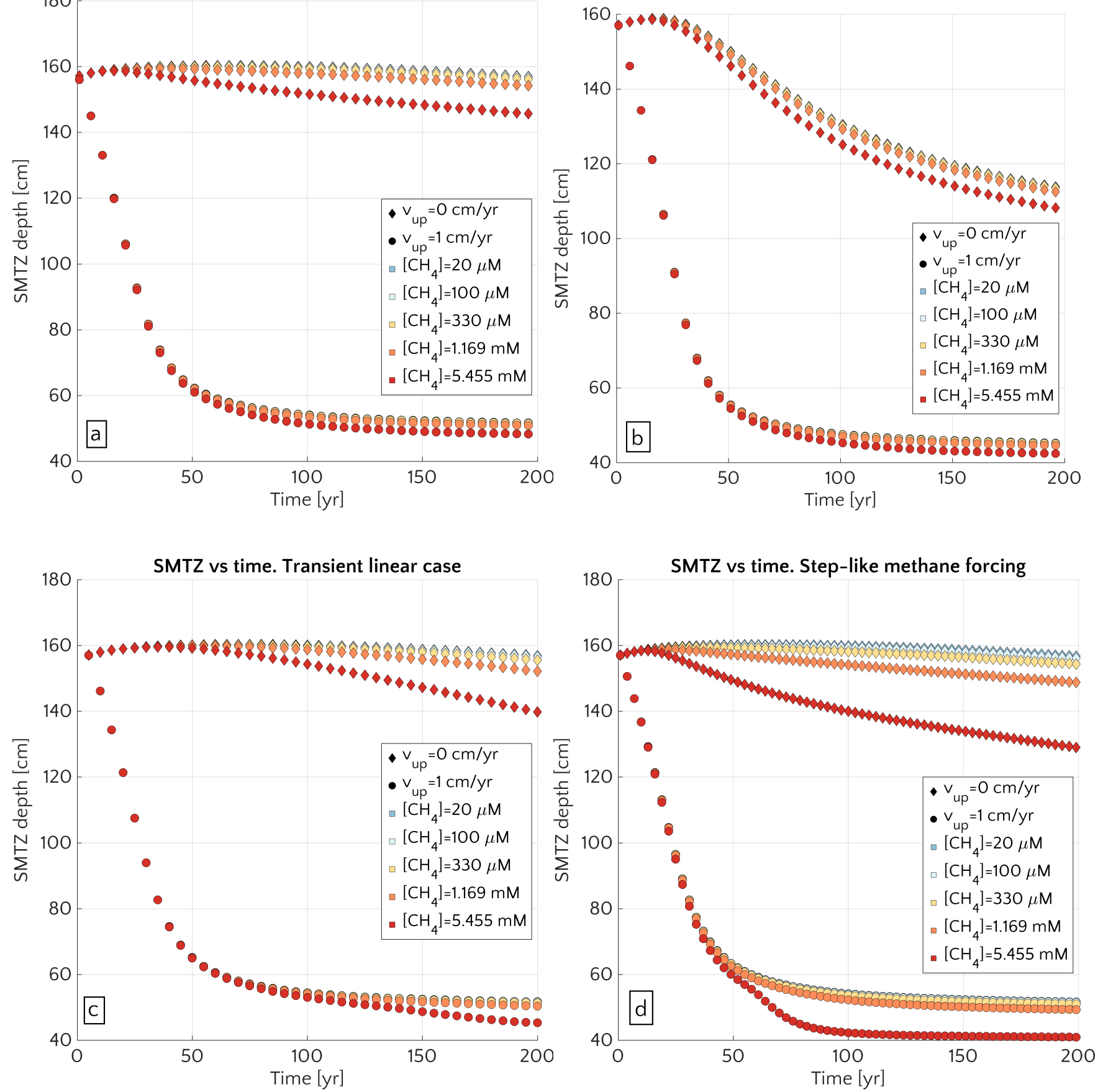

Figure S18. Movement of SMTZ over time for passive (diamonds) and active (circle) setups and different $\left[\mathrm{CH}_{4}\right]_{-} . a$. Seasonal methane forcing from below. $b$. Seasonal methane forcing from below and seasonal sulfate forcing from above. $c$. Linear methane forcing from below. c. Step-like methane forcing from below. 


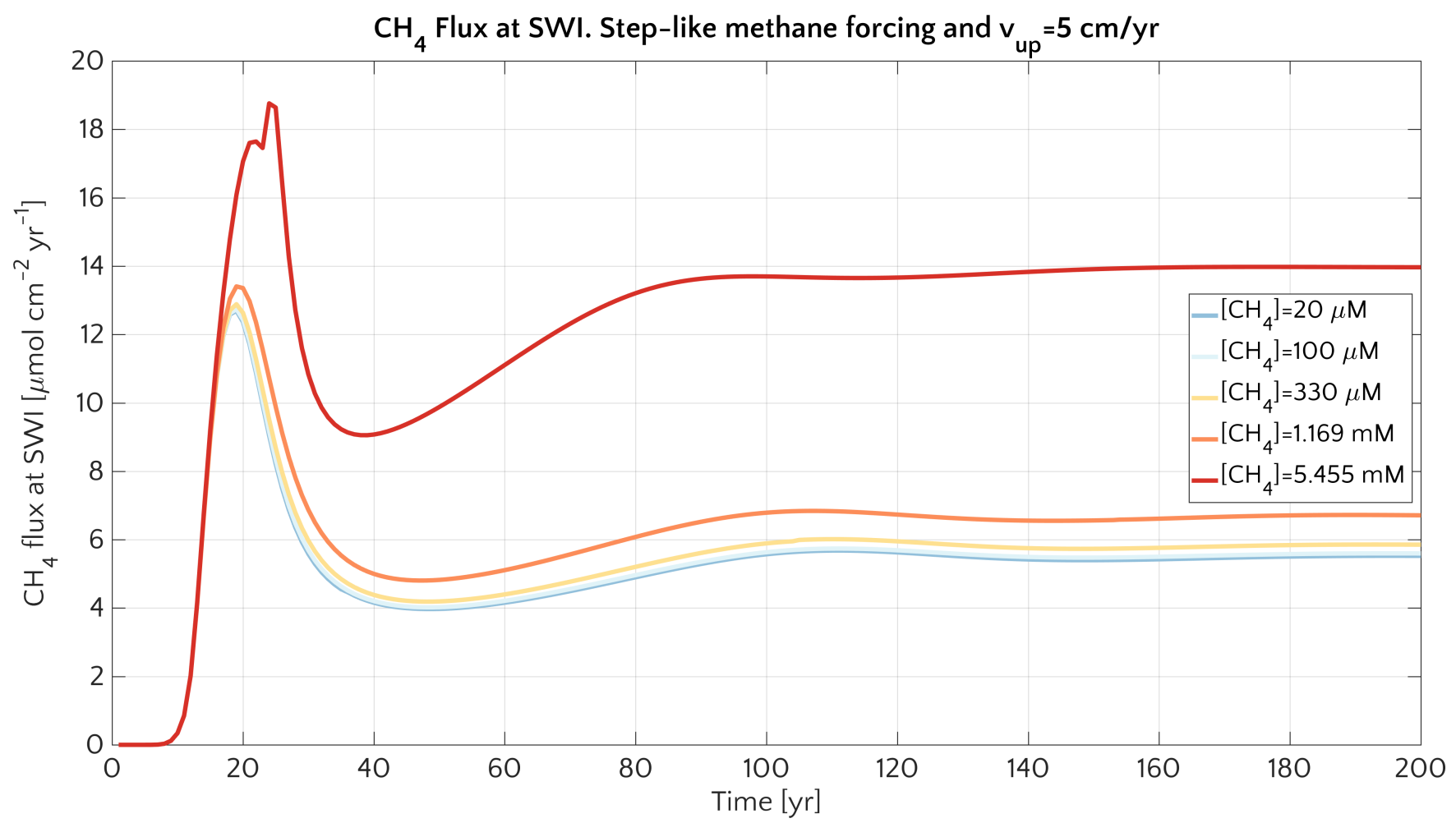

Figure S19. Flux of methane at SWI versus time for scenario 4 (step-like methane increase) and $v_{u p}=5 \mathrm{~cm} / \mathrm{yr}$. Colours identify simulations with different $\mathrm{CH}_{4}$ bottom concentrations. 


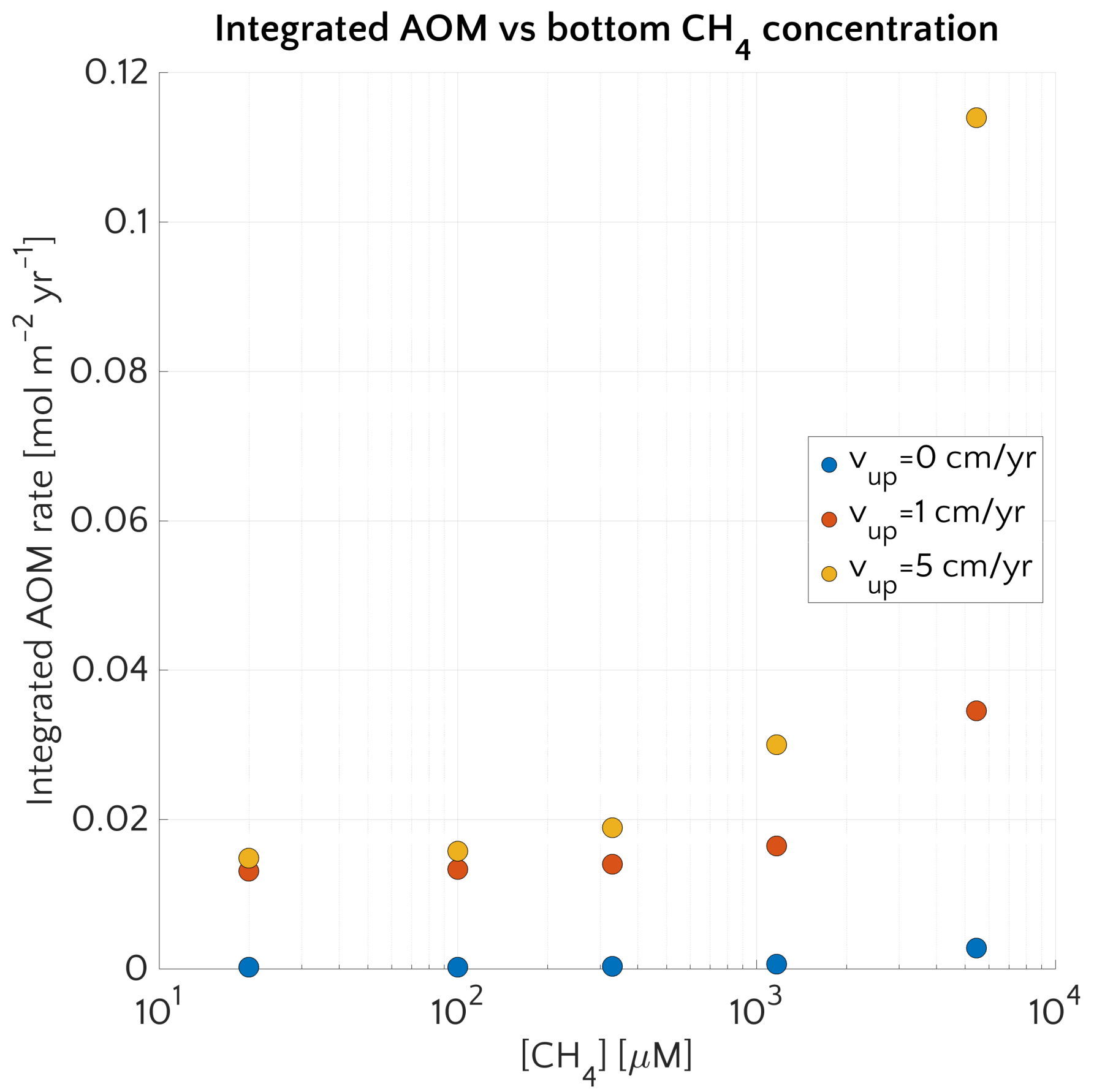

Figure S20. Vertically integrated AOM $v s$ the bottom methane concentration $\left[\mathrm{CH}_{4}\right]_{-}$for three different $v_{u p}$. 
Integrated biomass vs bottom $\mathrm{CH}_{4}$ concentration
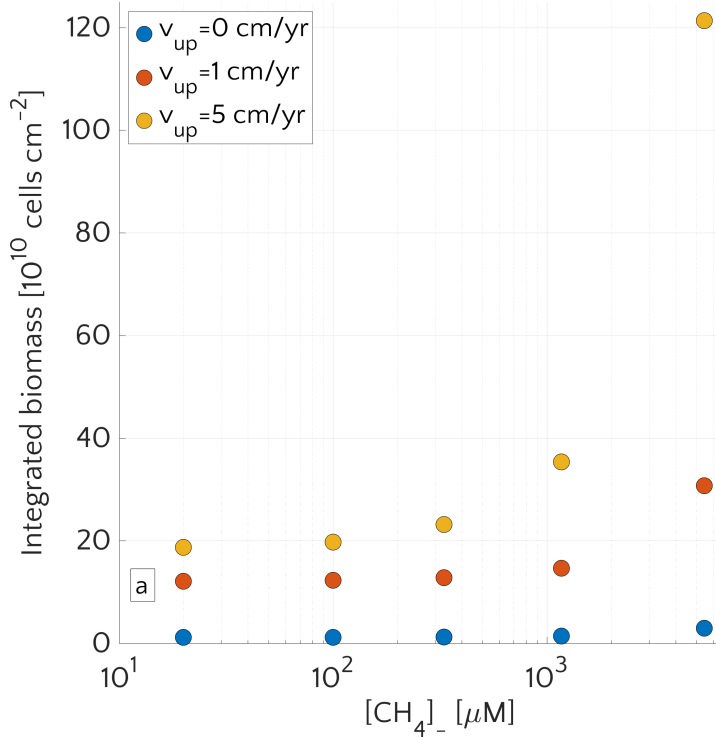

\section{Flux ratio vs bottom $\mathrm{CH}_{4}$ concentration}

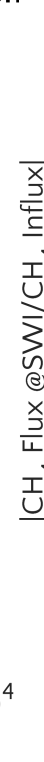

- $v_{\text {up }}=0 \mathrm{~cm} / \mathrm{yr}$

- $v_{\text {up }}=1 \mathrm{~cm} / \mathrm{yr}$

- $v_{\text {up }}=5 \mathrm{~cm} / \mathrm{yr}$

○

:

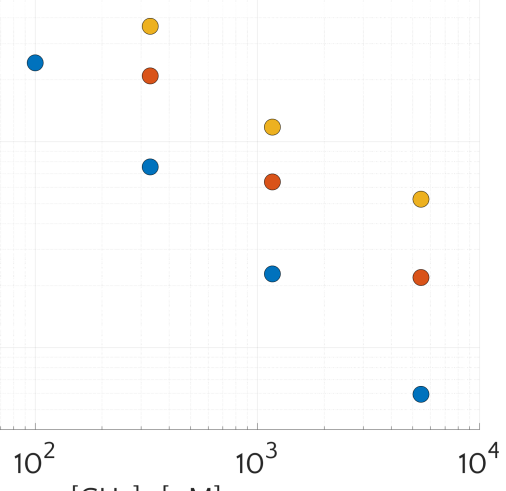

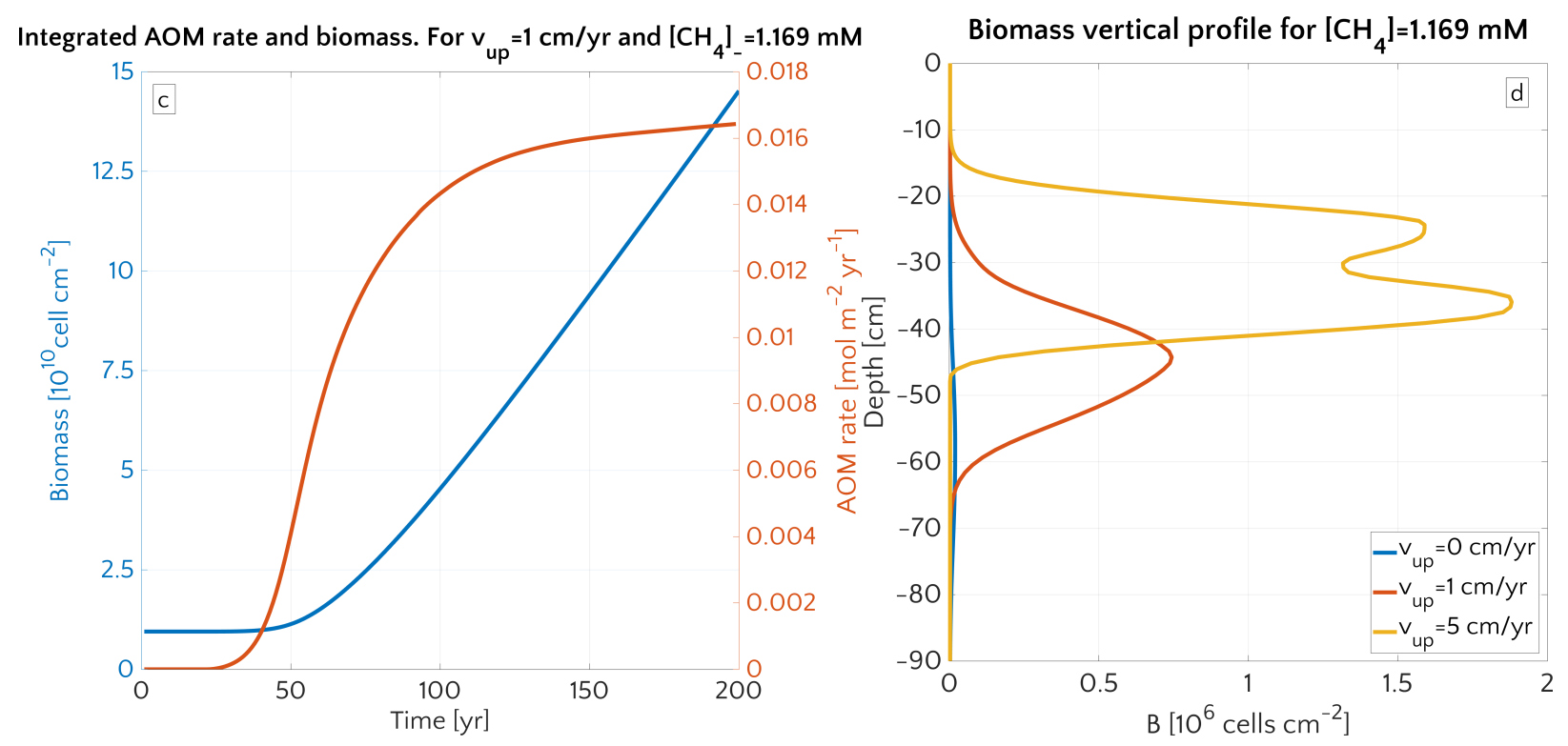

Figure S21. $a$. Vertically integrated biomass after 200 years $v s$ the bottom methane concentration $\left[\mathrm{CH}_{4}\right]_{-}$for three different $v_{u p} . b$. Absolute value of the ratio of the methane flux at the SWI to the advective methane flux at the base of sediment column vs the bottom methane concentration $\left[\mathrm{CH}_{4}\right]_{-}$for three different $v_{u p} . c$. Time evolution of the vertically integrated AOM biomass (blue) and vertically integrated AOM rate (red) for the step-like simulation with $\left[\mathrm{CH}_{4}\right]_{-}=1.169 \mathrm{mM}$ and $v_{u p}=1 \mathrm{~cm} / \mathrm{yr}$. $d$. AOM biomass vertical profile after 200 years for three different $v_{u p}$ in case of step-like simulation with $\left[\mathrm{CH}_{4}\right]_{-}=1.169 \mathrm{mM}$. 


\section{References}

Aller, R. C. and Blair, N. E.: Early diagenetic remineralization of sedimentary organic C in the Gulf of Papua deltaic complex (Papua New Guinea): net loss of terrestrial C and diagenetic fractionation of C isotopes, Geochimica et Cosmochimica Acta, 68, 1815-1825, 2004.

Arndt, S., Jørgensen, B. B., LaRowe, D. E., Middelburg, J., Pancost, R., and Regnier, P.: Quantifying the degradation of organic matter in marine sediments: a review and synthesis, Earth-science reviews, 123, 53-86, 2013.

Athy, L. F.: Density, porosity, and compaction of sedimentary rocks, AAPG Bulletin, 14, 1-24, 1930.

Bauch, H. A., Mueller-Lupp, T., Taldenkova, E., Spielhagen, R. F., Kassens, H., Grootes, P. M., Thiede, J., Heinemeier, J., and Petryashov, V.: Chronology of the Holocene transgression at the North Siberian margin, Global and Planetary Change, 31, 125-139, 2001.

Berg, P.: Dynamic Modeling of Early Diagenesis and Nutrient Cycling. A Case Study in an Artic Marine Sediment, American Journal of Science, 303, 905-955, 2003.

Berner, R. A.: Early diagenesis: a theoretical approach, 1, Princeton University Press, 1980.

Boudreau, B. P.: Diagenetic models and their implementation, vol. 505, Springer Berlin, 1997.

Boudreau, B. P. and Ruddick, B. R.: On a reactive continuum representation of organic matter diagenesis, American Journal of Science, 291, 507-538, 1991.

15 Brüchert, V.: Unpublished raw data, 2020.

Brüchert, V., Bröder, L., Sawicka, J. E., Tesi, T., Joye, S. P., Sun, X., Semiletov, I. P., and Samarkin, V. A.: Carbon mineralization in Laptev and East Siberian sea shelf and slope sediment, Biogeosciences, 15, 471-490, 2018.

Claypool, G. E. and Kaplan, I.: The origin and distribution of methane in marine sediments, in: Natural gases in marine sediments, pp. 99-139, Springer, 1974.

20 Dale, A. W., Regnier, P., and Van Cappellen, P.: Bioenergetic controls on anaerobic oxidation of methane (AOM) in coastal marine sediments: a theoretical analysis, American Journal of Science, 306, 246-294, 2006.

Dale, A. W., Aguilera, D., Regnier, P., Fossing, H., Knab, N., and Jørgensen, B. B.: Seasonal dynamics of the depth and rate of anaerobic oxidation of methane in Aarhus Bay (Denmark) sediments, Journal of Marine Research, 66, 127-155, 2008a.

Dale, A. W., Van Cappellen, P., Aguilera, D., and Regnier, P.: Methane efflux from marine sediments in passive and active margins: Estimations from bioenergetic reaction-transport simulations, Earth and Planetary Science Letters, 265, 329-344, 2008b.

Dale, A. W., Meyers, S. R., Aguilera, D. R., Arndt, S., and Wallmann, K.: Controls on organic carbon and molybdenum accumulation in Cretaceous marine sediments from the Cenomanian-Turonian interval including Oceanic Anoxic Event 2, Chemical Geology, 324-325, 28-45, 2012.

Dale, A. W., Nickelsen, L., Scholz, F., Hensen, C., Oschlies, A., and Wallmann, K.: A revised global estimate of dissolved iron fluxes from marine sediments, Global Biogeochemical Cycles, 29, 691-707, https://doi.org/10.1002/2014gb005017, https://doi.org/10.1002\% 2F2014gb005017, 2015.

Froelich, P., Klinkhammer, G., Bender, M. L., Luedtke, N., Heath, G. R., Cullen, D., Dauphin, P., Hammond, D., Hartman, B., and Maynard, V.: Early oxidation of organic matter in pelagic sediments of the eastern equatorial Atlantic: suboxic diagenesis, Geochimica et cosmochimica acta, 43, 1075-1090, 1979.

35 Garcia, H. E., Boyer, T. P., Locarnini, R. A., Antonov, J. I., Mishonov, A. V., Baranova, O. K., Zweng, M. M., Reagan, J. R., Johnson, D. R., and Levitus, S.: World Ocean Atlas 2009, Volume 3: Dissolved Oxygen, Apparent Oxygen Utilization, and Oxygen Saturation, 2010a.

Garcia, H. E., Locarnini, R. A., Boyer, T. P., Antonov, J. I., Baranova, O. K., Zweng, M. M., Reagan, J. R., Johnson, D. R., Mishonov, A. V., and Levitus, S.: World Ocean Atlas 2009, Volume 4: Nutrients (phosphate, nitrate, silicate), $2010 \mathrm{~b}$.

Glasby, G. P.: Manganese: predominant role of nodules and crusts, in: Marine geochemistry, pp. 371-427, Springer, 2006.

Han, P.: Characteristics and sedimentation rates at the shallow Laptev Sea, Master's thesis, University of Bremen, 2014.

5 LaRowe, D. E., Burwicz, E., Arndt, S., Dale, A. W., and Amend, J. P.: Temperature and volume of global marine sediments, Geology, 45, 275-278, 2017.

Meile, C. and Van Cappellen, P.: Particle age distributions and $\mathrm{O} 2$ exposure times: Timescales in bioturbated sediments, Global biogeochemical cycles, 19, 2005.

Middelburg, J. J., Soetaert, K., and Herman, P. M.: Empirical relationships for use in global diagenetic models, Deep Sea Research Part I: Oceanographic Research Papers, 44, 327-344, 1997

Millero, F. J.: Thermodynamics of the carbon dioxide system in the oceans, Geochimica et Cosmochimica Acta, 59, 661-677, https://doi.org/10.1016/0016-7037(94)00354-o, https://doi.org/10.1016\%2F0016-7037\%2894\%2900354-o, 1995.

Mucci, A.: The solubility of calcite and aragonite in seawater at various salinities, temperatures, and one atmosphere total pressure, American Journal of Science, 283, 780-799, https://doi.org/10.2475/ajs.283.7.780, https://doi.org/10.2475\%2Fajs.283.7.780, 1983.

15 Pauss, A., Andre, G., Perrier, M., and Guiot, S. R.: Liquid-to-gas mass transfer in anaerobic processes: inevitable transfer limitations of methane and hydrogen in the biomethanation process, Appl. Environ. Microbiol., 56, 1636-1644, 1990. 
Regnier, P., Dale, A. W., Arndt, S., LaRowe, D., Mogollón, J., and Van Cappellen, P.: Quantitative analysis of anaerobic oxidation of methane (AOM) in marine sediments: a modeling perspective, Earth-Science Reviews, 106, 105-130, 2011.

Sales de Freitas, F.: Deciphering the relationships between reactivity and sources of organic matter in marine sediments: a coupled large-scale model and lipid biomarker analysis, Ph.D. thesis, School on Earth Sciences, University of Bristol, 2018.

Stein, R. and Fahl, K.: Holocene accumulation of organic carbon at the Laptev Sea continental margin (Arctic Ocean): sources, pathways, and sinks, Geo-Marine Letters, 20, 27-36, https://doi.org/10.1007/s003670000028, https://doi.org/10.1007\%2Fs003670000028, 2000.

Stein, R., Boucsein, B., Fahl, K., de Oteyza, T. G., Knies, J., and Niessen, F.: Accumulation of particulate organic carbon at the Eurasian continental margin during late Quaternary times: controlling mechanisms and paleoenvironmental significance, Global and Planetary Change, 31, 87-104, 2001.

Strobl, C., Schulz, V., Vogler, S., Baumann, S., Kassens, H., Kubik, P., Suter, M., and Mangini, A.: Determination of depositional beryllium10 fluxes in the area of the Laptev Sea and beryllium-10 concentrations in water samples of high northern latitudes, in: Land-Ocean Systems in the Siberian Arctic, pp. 515-532, Springer, 1998.

Stumm, W. and Morgan, J. J.: Aquatic Chemistry: Chemical Equilibria and Rates in Natural Waters \{Environmental Science and Technology\}, Wiley, 1996.

Thullner, M., Dale, A. W., and Regnier, P.: Global-scale quantification of mineralization pathways in marine sediments: A reaction-transport modeling approach, Geochemistry, geophysics, geosystems, 10, 2009.

Van Cappellen, P. and Wang, Y.: Cycling of iron and manganese in surface sediments; a general theory for the coupled transport and reaction of carbon, oxygen, nitrogen, sulfur, iron, and manganese, American Journal of Science, 296, 197-243, 1996.

35 Wallmann, K., Aloisi, G., Haeckel, M., Obzhirov, A., Pavlova, G., and Tishchenko, P.: Kinetics of organic matter degradation, microbial methane generation, and gas hydrate formation in anoxic marine sediments, Geochimica et Cosmochimica Acta, 70, 3905-3927, 2006. 